\title{
Embedding of the Saddle Point of Index two on the PES of the Ring Opening of Cyclobutene
}

\author{
Wolfgang Quapp*, Josep Maria Bofill ${ }^{\dagger}$
}

July 2, 2015

\section{Abstract}

The ring opening of cyclobutene is characterized by a competition of the two different pathways: a usual pathway over a saddle of index one $\left(\mathrm{SP}_{1}\right)$ along the conrotatory behavior of the end groups, as well as a "forbidden" pathway over a saddle point of index two $\left(\mathrm{SP}_{2}\right)$ along the disrotatory behavior of the end $\mathrm{CH}_{2}$ groups. We use the system of ordinary differential equations for the method of the gentlest ascent dynamics (GAD) to determine saddle points of the potential energy surface (PES) of the ring opening of cyclobutene to cis-butadiene. We apply generalized GAD formulas for the search of a saddle point of index two.
*Mathematisches Institut, Universität Leipzig, PF 100920, D-04009 Leipzig, Germany, quapp@uni-leipzig.de

†Departament de Química Orgànica, Universitat de Barcelona, and Institut de Química Teòrica i Computacional, Universitat de Barcelona, (IQTCUB), Martí i Franquès, 1, 08028 Barcelona, Spain, jmbofill@ub.edu
To understand the relation of the different regions of the PES (around minimums, around SPs of index one or two) we also calculate valley-ridge inflection points (VRI) on the PES by using Newton trajectories (NT). VRIs and the corresponding singular NTs subdivide the regions of "attraction" of the different SPs. We calculate the connections of the $\mathrm{SP}_{2}$ (in its different symmetry versions) with different SPs of index one of the PES by different "reaction pathways". We compare the possibilities of the tool of the GAD curves for the exploration of PESs with these of Newton trajectories.

The barrier of the disrotatory $\mathrm{SP}_{2}$ is somewhat higher than the barrier of the conrotatory $\mathrm{SP}_{1}$, however, pathways across the slope to the $\mathrm{SP}_{2}$ open additional reaction valleys. 


\section{INTRODUCTION}

Mathematics has had a profound impact on science, providing a means to understand the world around us in unprecedented ways. ${ }^{1}$ With the advantage of the computer age, the subject of modelization has hugely grown in importance. In particular, over the last decades significant advances in our understanding of the world of chemistry have culminated in the development of a very large set of concepts. Some of these concepts are the potential energy surface (PES) ${ }^{2-4}$ and the chemical reaction path $(\mathrm{RP})^{5}$ being the basic concepts for the theories of chemical dynamics and chemical transformations. The PES is a continuous function of the coordinates of the nuclei over an $\mathbb{R}^{N}$, thus it is an $N$-dimensional hypersurface. It should have continuous derivatives up to a certain order for our treatments. If Cartesian coordinates are used, then $N=3 n$, however, we may also use $N=3 n-6$ nonredundant, internal coordinates for an $n$-atomic molecule.

The PES can be seen as formally divided in catchments associated with local minima. ${ }^{2}$ For such a picture one has in mind steepest descent (SD) curves. The first order saddle points or transition states (TSs) are located at the deepest points of the boundary of the basins. TSs and minima correspond to stationary points of the PES. Two adjacent minima of the PES can be connected through a TS via a continuous curve in the $N$-dimensional coordinate space, describing the coordinates of the nuclei. The curve characterizes a reaction path. The concept of the RP has found a widespread use in descriptions of reaction mechanisms, which are based on geometrical rearrangements of the atoms comprising a given chemical system. ${ }^{6}$ More specifically, an RP links the transformation from reactant to product to a path located in a multidimensional configuration space. The use of the configura- tion space instead of the phase space (position and momentum) obviously means the neglect of the contribution of the kinetic energy of the nuclei. Thus, the RP concept describes the chemical reactions in purely geometrical changes. But even with this limitation the $\mathrm{RP}$ concept is widely used within the chemical community.

In recent years, however, theoretical and experimental advances have allowed us to look more deeply into the dynamics of excited molecules, and there is growing recognition that a highly excited molecule is more like a wing-suit diver who sails over the PES, roaming to new valleys and sometimes landing on unexpected terrain. Such non-RP dynamics may well be the rule rather than the exception for hot molecules. ${ }^{7-11}$ Recently, theoretical work has suggested that a hint for many of these non- $\mathrm{RP}$ processes is a "second-index saddle point" $\left(\mathrm{SP}_{2}\right)$ on the PES. The index is the number of unstable directions attached to the saddle. In our usual imagination, an $\mathrm{SP}_{2}$ is a hilltop separating two mountain passes. But on an $N$-dimensional PES again it is a saddle with an (N-2)-dimensional ridge being orthogonal to the 2-dimensional col. An early pioneering work on saddle points of index 2 on PESs and their role in theoretical reactivity investigations was done by Heidrich and Quapp, ${ }^{12}$ and by Minyaev et al. ${ }^{13}$ In the last reference, the reaction coordinate for the isomerization of the $\mathrm{NH}_{5}$ complex was followed over an $\mathrm{SP}_{2}$. A more dynamical point of view is applied elsewhere. ${ }^{10}$ Pathways to reactants that find their way over the slope below an $\mathrm{SP}_{2}$ will undergo very different forces and dynamics and emerge with distinct products or product distributions. The importance of roaming is that such features on the potential energy landscape appear almost universally in the vicinity of simple bond fission processes in molecules. Although seen and documented clearly in small molecules, 
the related dynamics are perhaps suspected to play a role in normal molecules up to even protein folding. For this reason it is necessary to have methods to find saddle points of a higher index, especially of index 2 , to study the topography around these points to predict the dynamical behavior. Often already the slope down below an $\mathrm{SP}_{2}$ may open new pathways to other valleys. On the other hand, the existence of an SP of index 2 can be inferred under a roaming behavior of a reaction.

The PES exhibits bifurcations of the valley path in the vicinity of so-called valley-ridge inflection (VRI) points. Recently one has found out that dynamics in the vicinity of a VRI point on the PES can play an important role in determining the product ratio, in a highly dissipative regime. ${ }^{14}$ For this reason the existence and location of VRIs on the $\mathrm{PES}$ is also an important question.

This article has many different objectives. While many curves satisfy the concept of the $\mathrm{RP}$, in the present study we use the 'gentlest ascent dynamics' (GAD) pathways proposed by E and Zhou, ${ }^{15}$ and the Newton trajectories $(\mathrm{NT})^{16-18}$ as paths to locate not only TSs but also saddle points of index 2 as well as VRIs. The purpose is to use the concepts to predict bifurcations and from that the caution that standard theories like Transition State Theory can not be used to predict ratios and stereospecificity.

NTs generate other catchments in comparison to SD curves. There emerge singular NTs which lead to valley-ridge inflection (VRI) points, and which are a net of curves dividing families of regular NTs. The singular NTs are the borders of corresponding catchments. Regular NTs connect stationary points with an index difference of one.

A third kind of curves used are the SD curves. ${ }^{19,20}$ We study with them the struc- ture of the hilltop of interest.

The contents of this paper are as follows: In the next section we summarize the basic formulae of the two curves, namely, GAD and NT. The subsections explain the mathematical nature and the method of integration of each of these two curves. Then we describe the numerical methods used. In the main section we focus on the special problem of the ring opening of cyclobutene. This isomerization has been studied and reviewed several times in the last decades. The PES of the system is well known. However, we take the system with the purpose to study the capacity of the above mentioned methods, to explore the features of the PES and in particular around the region of the $\mathrm{SP}_{2}$ which is associated with the disrotatory process, and which may open the possibility of a roaming mechanism. If this is true, then the above methods can be used in a general form as a predictive tool for the possible existence of a roaming mechanism, before one starts a dynamic study. A last section concludes the paper. Finally in an appendix parts of the procedure of the present calculations are reported in detail.

\section{Review on the GAD and Newton Trajectory Pathways}

\section{The Theory of the GAD Curve}

\section{Basic of the GAD Model}

After its introduction by E and Zhou, ${ }^{15}$ the GAD path has been deeply analyzed. The name 'gentlest ascent' was coined in a very early paper by Crippen and Scheraga. ${ }^{21} \mathrm{~A}$ recent work due to Quapp and Bofill ${ }^{22}$ shows that the GAD pathway can be understood through the concept of the image potential function. ${ }^{23}$ The paper also narrows down 
this type of curve which sometimes does not describe an RP, because it can miss the TS. Other works doing the analysis and possibly use it as an $\mathrm{RP}$ are due to $\mathrm{Li}$ et al.,${ }^{24}$ to Samanta et al.,${ }^{25}$ and to Zeng et al. ${ }^{26}$

Let be $V(\mathbf{q})$ an $N$-dimensional surface over $\mathbb{R}^{N}$, let $\operatorname{grad}(\mathbf{q})$ be its gradient vector, $\nabla_{\mathbf{q}} V(\mathbf{q})$, and let $\mathbf{H}(\mathbf{q})$ be the matrix of its second derivatives, the Hessian $\nabla_{\mathbf{q}}$ grad $^{T}$. The reason for introduction trajectories by a 'gentlest ascent' dynamics (GAD) is the purposeful search for SPs of index one, in a first step, or for SPs of a higher, fixed index, for example for 'summits' of index two. The GAD curves are trajectories along the following system of $2 N$ coupled differential equations ${ }^{15,22}$

$q^{i \prime}=-\operatorname{grad}_{i}+2 \frac{\sum_{j} v^{j} \operatorname{grad}_{j}}{\sum_{k} v^{k} v^{k}} v^{i}, \quad i=1, \ldots, N$

$v^{i \prime}=-\sum_{k} H_{i k} v^{k}+\frac{\sum_{k} \sum_{j} v^{k} H_{k j} v^{j}}{\sum_{k} v^{k} v^{k}} v^{i}, \quad i=$

with the initial conditions

$$
\begin{gathered}
\mathbf{q}\left(t_{o}\right)=\mathbf{q}_{o} \\
\mathbf{v}\left(t_{o}\right)=\operatorname{grad}\left(t_{o}\right) .
\end{gathered}
$$

Cartesian coordinates are used. The meaning of the two first Eqs. (1) and (2) is the following: If $\mathbf{v}$ is an eigenvector of $\mathbf{H}$ then the right hand side of Eq.(2) is zero. Thus no change to $\mathbf{v}$ emerges. If additionally the eigenvector is the one which belongs to the smallest eigenvalue near the SP, and the gradient is parallel to this eigenvector, then the ascent to the SP in Eq.(1) is along this direction. This is the search along the SP-col and at the SP the gradient disappears, so the dynamical system converges there.

A shortform of the Eqs. (1) and (2) is the following. We define the projection operator to vector $\mathbf{v}$ by a dyadic product forming an $(N \times N)$-matrix

$$
\mathbf{P}_{\mathbf{v}}=\frac{1}{\sum_{k} v^{k} v^{k}}\left(v^{i} v^{j}\right)
$$

with entries for $i=1, \ldots, N, j=1, \ldots, N$. The GAD equations become

$$
\begin{aligned}
& \mathbf{q}^{\prime}=-\left(\mathbf{I}-2 \mathbf{P}_{\mathbf{v}}\right) \operatorname{grad}, \\
& \mathbf{v}^{\prime}=-\left(\mathbf{I}-\mathbf{P}_{\mathbf{v}}\right) \mathbf{H} \mathbf{v} .
\end{aligned}
$$

I is the unit matrix. Note that an acceleration of the GAD convergence can be found if one uses an additional factor by a trick of the Lanczos-Hestenes diagonalization technique $^{27,28}$ in Eq. (7)

$$
\mathbf{v}^{\prime}=-\left(\mathbf{H}_{D}-\frac{\mathbf{v}^{T} \mathbf{H} \mathbf{v}}{\mathbf{v}^{T} \mathbf{v}} \mathbf{I}\right)^{-1}\left(\mathbf{I}-\mathbf{P}_{\mathbf{v}}\right) \mathbf{H} \mathbf{v} .
$$

where $\mathbf{H}_{D}$ is a diagonal matrix, namely it is the diagonal part of the Hessian. This new term ean be seen as the precondition being the approximated inverse to the Jacobian of the functional related to the diagonalization problem, the Rayleigh-Ritz quotient. ${ }^{29,30}$

The analysis of the system(1) and (2) shows that the index one SPs are stable fixed points. ${ }^{15}$ Usually, if one starts near a minimum in the corresponding minimum well, then the dynamical system turns by itself the $\mathbf{v}$ vector to the smallest eigenvector; and we find convergence to a corresponding SP. But we have also reported another case for the example of the radical $\mathrm{CH}_{3} \mathrm{O}^{\bullet}{ }^{31}$ The behavior of the GAD concept is studied for the isomerization of the radical, $\mathrm{CH}_{3} \mathrm{O} \bullet$ to - $\mathrm{CH}_{2} \mathrm{OH}$, and its decomposition to $\mathrm{H}-\mathrm{C}^{\bullet}=\mathrm{O}$ $+\mathrm{H}_{2}$, either stepwise or concerted, see also elsewhere. ${ }^{32}$ Recently, an application of the GAD to Physical Chemistry is demonstrated for point defect activity on a surface. ${ }^{24,33} \mathrm{We}$ will apply GAD to a single molecule in the empty space. The description of the PES of 
an $n$-atomic molecule can be done in $3 n-6$ internal coordinates. They have to be nonredundant, however, they are curvilinear. The inclusion of the curvilinear metric of nonredundant internal coordinates is given in the next subsection. The application of GAD to SPs of a higher index is given below.

\section{The GAD Model with a Metric}

We assume $N=3 n-6$ nonredundant internal coordinates for an $n$-atomic molecule. Now the corresponding metric relations are used, ${ }^{18,20}$ because normally, the PES is represented in curvilinear coordinates. The calculations are done with the modified ansatz

$q^{i \prime}=-g^{i j} \operatorname{grad}_{j}+2 \frac{\left(v^{j} \operatorname{grad}_{j}\right)}{\left(v^{k} g_{k j} v^{j}\right)} v^{i}, \quad i=1, \ldots, N$

$v^{i \prime}=-g^{i j} H_{j k} v^{k}+\frac{\left(v^{k} H_{k j} v^{j}\right)}{\left(v^{k} g_{k j} v^{j}\right)} v^{i}, \quad i=1, \ldots, M$

Here, $\left(g_{k j}\right)$ is the covariant metric matrix, but $\left(g^{i j}\right)$ is the contravariant one. In contrast to Eqs.(1) and (2) we here use the summation convention: over a double index, an upper and lower one, we have to sum it up. Thus when an index variable appears twice in a single term it implies summation of that term over all the possible values of the index. The summation over a set of indexed terms in a formula is used to achieve notational brevity. Note that the upper indices are not exponents but are indices depicting a contravariant vector or tensor. Here we assume that $\mathbf{q}$ and $\mathbf{v}$ are contravariant vectors, however grad is a covariant vector, and $\mathbf{H}$ is (an approximation) of a twofold covariant tensor (at least near the SPs of the PES). ${ }^{18,34}$ Thus, we can form the scalar products $v^{j} \operatorname{grad}_{j}$ in Eq. (1), as well as $v^{k} H_{k j} v^{j}$ in Eq. (2), but for the norm in the denominator in Eqs.(1) and (2) we have to include the metric matrix. Also for the first summand on the right hand side, the gradient component, we have to 'change' the character to adapt it to the left hand side vector using the inverse metric matrix in Eq. (9). This happens formally also for the vector $\left(H_{k j} v^{j}\right)$ in Eq. (10) which is covariant like the gradient.

Eq. (10) contains the (contravariant) eigenvector problem for the covariant tensor $\mathbf{H}$ : To make the characters equal, we have to treat the problem for the eigenvalue $\lambda$

$g^{i k} H_{k j} v^{j}=\lambda v^{i}$ or the inverse $H_{r j} v^{j}=\lambda g_{r i} v^{i}$.

Then follows in Eq. (10) that for such an eigenvector $\mathbf{v}$ the steplength becomes zero. Contrary in Eq. (9), if $\mathbf{v}$ becomes parallel to $\mathrm{N}$ the contravariant gradient vector $g^{i j} \operatorname{grad}_{j}=$ $k v^{i}$, with a constant $k$, or the covariant component of $\mathbf{v}$ becomes parallel: $\operatorname{grad}_{j}=k g_{i j} v^{i}$, $N$ (which is the usual behavior on the col at the SP) then the step becomes $k v^{i}$ along the corresponding eigenvector with steplength $k=$ (norm of the gradient), thus it converges along the valley to the SP. From the point of view of Algebra, Eq. (9) is the 'mirror' transformation of the gradient to a plane which is orthogonal to the $\mathbf{v}$-direction.

\section{Search of SP's of Higher Index by GAD Trajectories}

Chemists have assumed over a long time that computations based on features (usually critical points, such as minima and first index SPs) of the PES provide enough predictions for relative rates associated with competing reactive channels, temperature dependence of reaction rates, branching ratios, etc. As noted in the introduction systems for which the standard RP picture is not valid include the growing class of the so-called non-RP reactions, and "roaming" mechanisms. The dynamics of these reactions is not mediated by a single conventional transition state associated with an SP of index one..$^{711-13,35-40}$ For 
this reason it is important to have a method that explores a region of a PES where an $\mathrm{SP}_{2}$ emerges. The GAD method is a potential algorithm to be used for this aim. Now we use the ansatz reported in Ref. 22, but modified by the nonlinear metric tensors

$$
q^{i \prime}=-g^{i j} \operatorname{grad}_{j}+2 \frac{\left(v^{j} \operatorname{grad}_{j}\right)}{\left(v^{k} g_{k j} v^{j}\right)} v^{i}+2 \frac{\left(w^{j} \operatorname{grad}_{j}\right)}{\left(w^{k} g_{k j} w^{j}\right)}
$$

$$
\begin{gathered}
v^{i \prime}=-g^{i j} H_{j k} v^{k}+\frac{\left(v^{k} H_{k j} v^{j}\right)}{\left(v^{k} g_{k j} v^{j}\right)} v^{i} \\
w^{i \prime}=-g^{i j} H_{j k} w^{k}+\frac{\left(w^{k} H_{k j} w^{j}\right)}{\left(w^{k} g_{k j} w^{j}\right)} w^{i},
\end{gathered}
$$

always for $i=1, \ldots, N$. It is a system of $3 N$ differential equations. The initial values are

$$
\mathbf{v}_{o}=\operatorname{grad}\left(t_{o}\right), \quad \mathbf{w}_{o} \perp \mathbf{v}_{o},
$$

where $\mathbf{w}_{o}$ is not unique. The usefulness of such a system of equations becomes apparent with its success: The two help directions $\mathbf{v}$ and $\mathbf{w}$ are now used analogously to search a 2-dimensional summit with its two negative eigendirections of the $\mathbf{H}$ matrix. The idea is from the Lanczos diagonalization procedure which is a well established diagonalization algorithm, see Ref. 41 and references therein in respect of the numerical problems. Note, that sometimes this $\mathrm{GAD}_{2}$ does not converge to an index two SP if on the pathway the vector $\mathbf{w}$ turns into the direction of $\mathbf{v}$. The two equations, namely, Eq. (13) and (14), are uncoupled; sometimes they do not lead to correct orthogonal vectors $\mathbf{v}$ and $\mathbf{w}$. This is avoidable if one uses at every step a Schmidt reorthogonalization (SO) of $\mathbf{w}$ against $\mathbf{v}$. Results are discussed in the main section below.

A more sophisticated ansatz is the following: We define the projection operator, including the metric, to vector $\mathbf{v}$ by a dyadic product forming an $(N \times N)$-matrix

$$
\mathbf{P}_{\mathbf{v}}=\frac{1}{\left(v^{k} g_{k r} v^{r}\right)}\left(v^{i} v^{j}\right)
$$

with entries for $i=1, \ldots, N, j=1, \ldots, N$. Be $\mathbf{G}^{-1}$ the inverse metric matrix $\left(g^{i j}\right), \mathbf{G}$ the usual metric matrix $\left(g_{i j}\right)$, and $\mathbf{I}$ be the

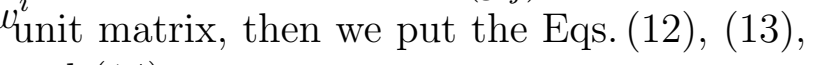
and (14) to

$$
\begin{gathered}
\mathbf{q}^{\prime}=-\left(\mathbf{G}^{-1}-2 \mathbf{P}_{\mathbf{v}}-2 \mathbf{P}_{\mathbf{w}}\right) \operatorname{grad}(\mathbf{q}) \\
\mathbf{v}^{\prime}=-\left(\mathbf{G}^{-1}-\mathbf{P}_{\mathbf{v}}\right) \mathbf{H}(\mathbf{q}) \mathbf{v}
\end{gathered}
$$

and $\mathbf{w}^{\prime}=$

$$
\begin{gathered}
-\left(\mathbf{G}^{-1}-\mathbf{P}_{\mathbf{w}}\right)\left(\mathbf{I}-\mathbf{P}_{\mathbf{v}} \mathbf{G}\right) \mathbf{H}(\mathbf{q})\left(\mathbf{I}-\mathbf{P}_{\mathbf{v}} \mathbf{G}\right) \mathbf{w} \\
+S O,
\end{gathered}
$$

where again $S O$ implies a Schmidt orthogonalization in the metric sense to the previous vector, v. Correspondingly the initial vectors Eq. (15) have to be orthogonal with

$$
v^{i}\left(t_{o}\right) g_{i j}\left(t_{o}\right) w^{j}\left(t_{o}\right)=0
$$

and the value for the Rayleigh-Ritz relation for $\mathbf{v}$

$$
\lambda_{v}(\mathbf{q})=\frac{\left(v^{k} H_{k j} v^{j}\right)}{\left(v^{k} g_{k j} v^{j}\right)}
$$

has to be in relation to that of $\mathbf{w}$ and to the eigenvalues, $e_{v_{1}}, e_{v_{2}}$ and $e_{v_{3}}$, by $e_{v_{1}} \leq \lambda_{v}(\mathbf{q}) \leq e_{v_{2}} \leq \lambda_{w}(\mathbf{q}) \leq e_{v_{3}}$ for every current point $\mathbf{q}$ of the $\mathrm{GAD}_{2}$ trajectory. In diverse tests we found sometimes cases where these relations are not always fulfilled; however, along the pathway they usually stabilize by themselves and converge with the correct relation of $\mathbf{v}$ and $\mathbf{w}$.

If one searches SPs of a still higher index $s$, the system is to extend correspondingly up to the $(s+$ first $)$ line for the vectors $\mathbf{v}_{1}=\mathbf{v}, \mathbf{v}_{2}=\mathbf{w}, \ldots$ to $\mathbf{v}_{s}$ by 


$$
\begin{gathered}
\mathbf{v}_{s}^{\prime}=-\left(\mathbf{G}^{-1}-\mathbf{P}_{\mathbf{v} s}\right) \\
\left(\mathbf{I}-\sum_{r=1}^{s-1} \mathbf{P}_{\mathbf{v} r} \mathbf{G}\right) \\
+S O \\
+\mathbf{H})\left(\mathbf{I}-\sum_{r=1}^{s-1} \mathbf{P}_{\mathbf{v} r} \mathbf{G}\right) \mathbf{v}_{s}
\end{gathered}
$$

A final remark on the above set of equations to the generalized GAD method:

If we are interested in the location of a stationary point of index $N$ where the number is the maximum of the dimension of the space then the generalized GAD equations are reduced to the steepest ascent equation. The proof is trivial, we take either Eq. (9) or Eq. (13), $q^{i \prime}=-g^{i j} \operatorname{grad}_{j}+2 \frac{\left(v_{1}^{j} \operatorname{grad}_{j}\right)}{\left(v_{1}^{k} g_{k j} v_{1}^{j}\right)} v_{1}^{i}+$

$$
\begin{aligned}
& 2 \frac{\left(v_{2}^{j} \operatorname{grad}_{j}\right)}{\left(v_{2}^{k} g_{k j} v_{2}^{j}\right)} v_{2}^{i}+\ldots+2 \frac{\left(v_{n}^{j} \operatorname{grad}_{j}\right)}{\left(v_{n}^{k} g_{k j} v_{n}^{j}\right)} v_{n}^{i} \\
& =-\left(g^{i j}-2 g^{i j}\right) \operatorname{grad}_{j}=g^{i j} \operatorname{grad}_{j} .
\end{aligned}
$$

In Eq. (23) the resolution of identity is used. The generalized GAD method to locate a stationary point of index $N$ is independent of the set of linear independent $\mathbf{v}$-vectors, $\left\{\mathbf{v}_{i}\right\}_{i=1}^{N}$.

\section{The Theory of the Newton Tra- jectories}

A special further definition of an RP is the reduced gradient following (RGF) ${ }^{42-49}$ and its equivalent definition, the so-called Newton trajectory (NT). ${ }^{50,51}$ It can be calculated by an Euler-Branin-step method following along the direction of the vector field $\mathbf{A}$ grad of the so called Branin differential equation ${ }^{52}$

$$
\frac{d \mathbf{q}(t)}{d t}= \pm \mathbf{A}(\mathbf{q}(t)) \operatorname{grad}(\mathbf{q}(t))
$$

where $\mathbf{A}$ is the adjoint matrix ${ }^{43}$ to the Hessian of the PES, its desingularized inverse, and grad is the gradient of the PES. $t$ is a curve length parameter, $\mathbf{q}$ is the current point. Matrix A is defined as $\left((-1)^{i+j} m_{i j}\right)^{T}$ where $m_{i j}$ is the minor of $\mathbf{H}$ obtained by deletion of the $i^{\text {th }}$ row and the $j^{\text {th }}$ column from $\mathbf{H}$, and taking the determinant. We use again $(3 n-6)$ non-redundant, internal coordinates, ${ }^{53}$ for the metric. A second definition ${ }^{s}$ of the NT is given by the projector equation

$$
\mathbf{P}_{\mathbf{r}} \operatorname{grad}(\mathbf{q}(t))=\mathbf{0} \text {. }
$$

The projector can be defined by a dyadic product with a normalized search direction, $\mathbf{r}$

$$
\mathbf{P}_{\mathbf{r}}=\mathbf{I}-\mathbf{r} \mathbf{r}^{T}
$$

where $\mathbf{I}$ is the unit matrix. The curve can be calculated numerically by the derivative of the projector equation along the curve parameter $t$ giving also the tangent of the NT. ${ }^{44}$ Eq. (25) means that the gradient points into the r-direction along an NT.

Stationary points of the PES have a zero gradient. Thus, NTs into all directions can start from here if one selects the sign of Eq. (24) according to the index of the stationary point. ${ }^{43,44,54}$ This means that the search direction of an NT, r, is optional at SPs. This is the reason why stationary points are attractive points for NTs from all directions. An SP of index 2 on a PES is also the crossing point of infinitely many NTs. It is an attractor if we take the positive sign in Eq. (24).

The index theorem ${ }^{55,56}$ that a regular NT (without a VRI point) cannot connect two stationary points of the same index holds. It connects stationary points with an index difference of one. Only a singular NT through a VRI connects, for example, a minimum and an SP of index two. The singular NT bifurcates at the VRI, and its other two branches can connect two SPs of index one. A method of the search of VRI points is described elsewhere. ${ }^{57}$

If $N>2$ then a singular NT as a curve does not divide the $\mathbb{R}^{N}$ into two distinct regions. Note that the $\mathbb{R}^{3}$ can be divided by 
a plane or a two-dimensional surface, but not by a one-dimensional curve. However, the net of the singular NTs on the PES divides the corresponding space into distinct regions; see an example represented in Fig. 7 in ref. 17. The regular NTs around a singular NT show a characteristic curvilinear behavior which causes their spatial division. Their curvilinear pattern near the singular NT is exploited in the method to approximate VRI points. ${ }^{57}$

\section{Numerical Methods and Computational Details}

The GAD equations are of a form that is amenable to direct numerical solution, and so the calculation of the GAD trajectories is done with a high order Runge-Kutta method (RK) as in refs. 22,31. The performance of such methods is very good and they converge as fast as possible to a correct solution. The step length along the pathway is automatically selected; of course, this usually costs some additional effort. However, on the other hand, in some regions the step length becomes large; and one avoids unnecessary steps. The method of choice here is the explicit Runge-Kutta method of order $8(5,3),{ }^{58}$ see subroutine DOP853. If we are interested in exact pathways then we use this very exact procedure. This often results a large number of steps. The same reason holds for not working with updates of the Hessian. In an Appendix, we report a part of the scratch file for the communication of the GAD and the GamessUS ${ }^{59}$ parts of the calculation.

A method for VRI points is described in Ref. 57. If a VRI is found, then a method with Euler-Branin-steps following along the vector field A grad gives an approximation of the corresponding singular NT. To visualize the result we use below the method of projections of the NT down from the full space of 24 internal coordinates into a plane. To draw the pictures we apply the Mathematica program ${ }^{60}$ and the Molden ${ }^{61}$ tool. For comparison, the eigenvalues and eigenvectors of the Hessian in internal coordinates (see Eqs.(11)) are calculated by an own program, at every step.

For the construction of the PES associated with the isomerization of cyclobutenebutadiene, the GamessUS program is used. ${ }^{59,62}$ We use the firefly code of A.A. Granovsky. ${ }^{63}$ Every GamessUS run is started with the current point in internal coordinates. We use from the output file the energy, the gradient, and the Hessian of the PES, as well as the metric matrices. For reasons explained below, the wave-function theory is used. It is well established that in the language of the wave-function theory the main features of a PES related to a molecular system are achieved using energy functionals that mainly incorporate the strong electron correlation, like RHF and its extension, the multiconfigurational SCF like CASSCF. ${ }^{64}$ It is assumed that the weak electron correlation only modifies the energy barriers between minimums and transition states. Because in the present work we are interested in the topography of the PES we will use energy functionals that take into account the strong electron correlation. Throughout the article the basis set is the $6-31 \mathrm{G}$ of Pople et al. ${ }^{65}$ 
The Topography of the Cyclobutene Isomerization to Butadiene Potential Energy Surface Revisited

\section{The Conrotatory Ring-Opening of Cyclobutene}

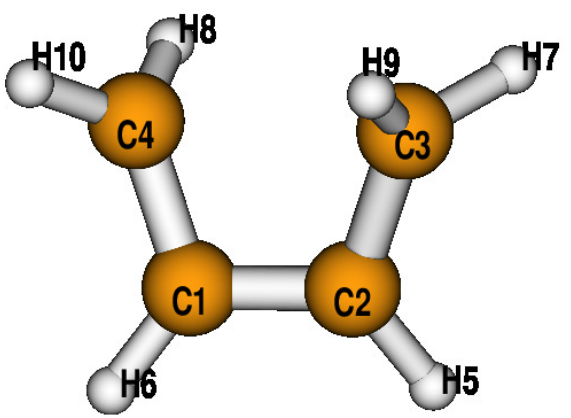

Figure 1: Minimum structure of cyclobutene predicted by the MCSCF model

\begin{tabular}{rrrrrrrr}
\hline $\mathrm{C}$ & & & & & & & \\
$\mathrm{C}$ & 1 & 1.329 & & & & & \\
$\mathrm{C}$ & 2 & 1.556 & 1 & $\operatorname{ccc} 394.6$ & & \\
$\mathrm{C}$ & 1 & 1.519 & 2 & $\operatorname{ccc} 496.1$ & 3 & dihc & 0.0 \\
$\mathrm{H}$ & 2 & 1.070 & 1 & 133.6 & 3 & & -180.0 \\
$\mathrm{H}$ & 1 & 1.070 & 2 & 132.8 & 4 & & 180.0 \\
$\mathrm{H}$ & 3 & 1.082 & 2 & 115.8 & 5 & $\operatorname{dih} 3$ & 65.4 \\
$\mathrm{H}$ & 4 & 1.082 & 1 & 116.1 & 6 & $\operatorname{dih} 4$ & -65.2 \\
$\mathrm{H}$ & 3 & 1.082 & 2 & 115.8 & 5 & $\operatorname{dih} 5$ & -65.4 \\
$\mathrm{H}$ & 4 & 1.082 & 1 & 116.1 & 6 & $\operatorname{dih} 6$ & 65.2 \\
\hline
\end{tabular}

Table 1: z-matrix for cylobutene minimum. Its MCSCF energy is -154.870 8 au.

Pericyclic and electrocyclic reactions play an important part in the realm of organic chemistry. The mechanisms of these reactions have been the subject of research in the past years, theoretical and experimental, and they are also the subject of interesting controversies. In the particular case of electrocyclic reactions, it is well known that the 3,4-disubstituted cyclobutene thermally rearranges a ring-opening to disubstituted butadiene. In principle, four different products are found, namely, the $(E, E-),(E, Z-)$, $(Z, E-),(Z, Z-)$ isomers. From a historical point of view at the early sixties, the mechanistic explanation used for these reactions was based in nature in steric effects which determine the stereochemical course of such a process.

The interconversion of cyclobutene to 1,3butadiene was discovered early, however, the studies of the mechanism were undertaken until the middle of the twenty century. In 1958, Walters reported the thermokinetic experimental data of the conversion of cyclobutene to butadiene. ${ }^{66}$ In this study Walters and coworkers concluded that the process is unimolecular, and it occurs at $150^{\circ} \mathrm{C}$. At the same time Vogel ${ }^{67}$ and Criegee and coworkers ${ }^{68,69}$ reported stereochemical studies of the ring-opening of the 3,4-disubstituted cyclobutenes leading to a considerable understanding of the mechanism.

The mechanism was considered as a concerted simultaneous bond breaking of the $\sigma$ bond $C_{3}-C_{4}$ and the $\pi$-bond $C_{1}=C_{2}$. The substituents located on each carbon of the $\sigma$-bond were required to move in the same sense, clockwise or counterclockwise in respect of the C-ring. This was the main conclusion of Srinivasan. ${ }^{70}$ In this manner the conrotatory motion of the substituents was assumed and understood, but not defined in a clear way.

In the beginning sixties of the last century it was assumed that the symmetry of the occupied Hartree-Fock orbitals, in particular the HOMO (highest occupied molecular orbital), is related to the stereochemistry of the cyclization during the reaction process. The 


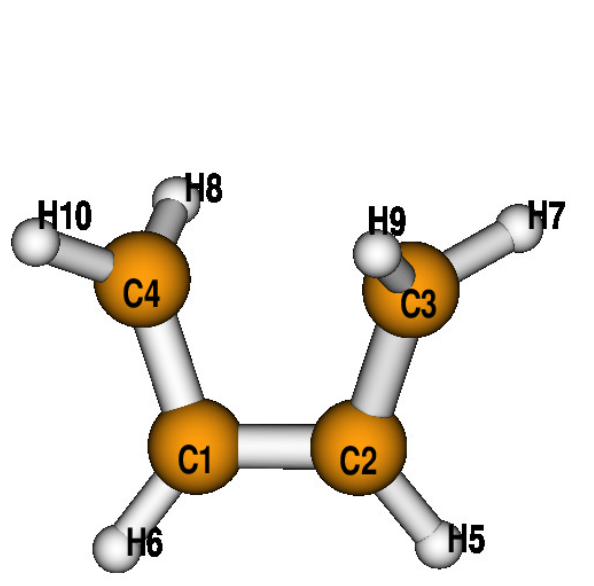

Figure 2: $\quad \mathrm{SP}_{1}$ for the conrotatory ring opening of the cyclobutene to cis-butadiene, named $\mathrm{SP}_{1 \text { ro }}$, cf. Table 2 .

\begin{tabular}{rrr}
\hline 1.342 & & \\
1.473 & 107.8 & \\
1.446 & 108.7 & 16.9 \\
1.074 & 127.9 & 180.1 \\
1.074 & 127.2 & 180.9 \\
1.078 & 122.3 & 39.3 \\
1.072 & 118.5 & -117.9 \\
1.072 & 117.7 & -115.6 \\
1.078 & 122.3 & 37.9 \\
\hline
\end{tabular}

Table 2: Coordinates of an $\mathrm{SP}_{1 \text { ro }}$ for the ring opening of the cyclobutene to cis-butadiene. See Table 1 for the z-matrix. The energy is -154.8189 au.

concept of the conservation of the molecular orbital-symmetry, the fundament of the Woodward-Hoffmann theory of electrocyclic reactions, ${ }^{71,72}$ provides an explanation of the electrocyclic cyclobutene-butadiene rearrangement. The set of occupied orbitals of the cyclobutene must correlate with the set of occupied orbitals of the product. Each one of these sets is used to build the Hartree-Fock configuration of reactant and product. This correlation is the graphic tool of the Woodward-Hoffmann theory applicable to any electrocyclic reaction. ${ }^{71-73}$ For this reason the RHF wave function is

\begin{tabular}{rrr}
\hline 1.473 & & \\
1.328 & 125.5 & \\
1.326 & 125.4 & 35.57 \\
1.076 & 115.5 & 179.0 \\
1.073 & 115.6 & 179.0 \\
1.093 & 121.3 & 2.0 \\
1.074 & 122.4 & -177.0 \\
1.074 & 122.4 & -177.0 \\
1.093 & 121.3 & 2.0 \\
\hline
\end{tabular}

Table 3: Coordinates of the nonplanar cisbutadiene minimum. Note the dihc= $C_{4} C_{1} C_{2} C_{3}=36^{\circ}$. See Table 1 for the zmatrix. The energy is $-154.8912 \mathrm{au}$.

sufficient to describe the region of the PES associated with the conrotatory evolution. The structure of the transition state for this process is reported in Fig. 2 (see also Table 2). The result has been reported several times by several authors, see e.g. Ref. 74,75 for more details and references therein, and the recent study of Koval et al. ${ }^{76}$

Finally, it is important to emphasize that the cyclobutene and the cis-butadiene in the conrotatory process by itself preserve the $C_{2}$ symmetry throughout the reaction, and the corresponding symmetry of each occupied orbital of the cyclobutene correlates with the occupied orbital of the cis-butadiene within this symmetry. Due to this fact the thermal conversion of cyclobutene to cis-butadiene proceeds in a conrotatory motion since in this way the lobes of the occupied orbitals involved in the reaction process rotate in the same direction, preserving the bonding character of all occupied orbitals. 


\section{The Disrotatory Ring-Opening of Cyclobutene. A Process through a non-Reaction Path}

Dewar $^{77,78}$ and Zimmerman ${ }^{79}$ classified the transition state of the conrotatory process as "aromatic" since it is, from an electronic point of view, equivalent to a Möbius array of four electrons. Using this terminology, the transition state of the disrotatory process is an equivalent to a Hückel array of four electrons from an electronic point of view, and due to this fact, it is "antiaromatic". For this reason the disrotatory process is higher in activation energy than the conrotatory process. This concept is exclusively based on the energy criterion and upon the existence of both transition states.

As mentioned above, the conrotatory process may be described correctly with a singleconfiguration wave function, let us say the Hartree-Fock configuration, since it represents an internal rearrangement of the orbitals taking place during the reaction. However, the description of the disrotatory process requires at least a two-configuration wave function involving both, the HOMO and the LUMO (lowest unoccupied molecular orbital). In this case the CASSCF wave function is very appropriate. A deeper question is related to the dimension and nature of the active space that permits to build the 'best' wave function for this reaction, in other words, the active space should be correctly selected. If one takes into account that during the process the HOMO and the LUMO are involved, one concludes that the appropriate minimum active space should be characterized by two electrons and two orbitals, these being the bonding and anti-bonding orbitals related to the $\mathrm{C} 3-\mathrm{C} 4$ bond of the cyclobutene to be broken during the reaction. For this reason the wave-function used is a CASSCF $(2,2)$ which is a function of two con- figurations.

The location and characterization of the stationary points for both conrotatory and disrotatory processes using the twoconfiguration wave function was given by Breulet and Schaefer. ${ }^{80}$ The conclusion of this computational study was that whereas the SP for the conrotatory process is a genuine transition state, for the disrotatory process it is a maximum in respect of two internal nuclear degrees of freedom (an index-two $\mathrm{SP}, \mathrm{SP}_{2}$ ). In the latter case, one of the internal nuclear degrees of freedom corresponds to the expected disrotatory displacement, however, the other demonstrates a symmetry breaking motion. An important remark emerges from the results of Breulet and Schaefer: ${ }^{80}$ in the PES of the ground electronic-state only the reaction path of the conrotatory isomerization exists, but a reaction path does not exist for the disrotatory process. Thus, as mentioned in the introduction and in a further subsection, for the disrotatory evolution the standard $\mathrm{RP}$ picture is not valid and the pathway should be included in the class of non-RP reactions, and may occur through a "roaming" mechanism. ${ }^{39}$ In other words, for the bulk of trajectories which characterize the dynamics, the average of the positions does not match a curve associated with an SP of index one.

From the electronic point of view we recall the terminology of Dewar ${ }^{77,78}$ and Zimmerman ${ }^{79}$ saying that for the ground state of the PES only the "aromatic" stationary point is a transition state and belongs to the concerted motion. The "antiaromatic" stationary point is not a TS of index one. A deeper electronic analysis of the "antiaromatic" region of the PES leads to a configuration interaction (CI) wave function. It reveals that the stationary point results from an avoided crossing between the ground and the doubly excited states of the sys- 
tem. ${ }^{81,82}$ For this reason, a wave function for this process should be constructed at least with two configurations, the Hartree-Fock and the resulting diexcited, to get a correct description of the disrotatory region of the PES. The wave-function $\operatorname{CASSCF}(2,2)$ satisfies these requirements as explained. See also again the review due to Houk. ${ }^{74}$

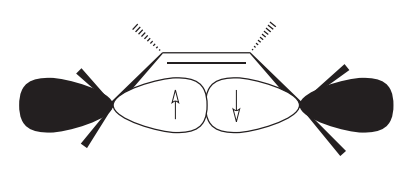

$\sigma-\mathrm{C}-\mathrm{C}$ orbital

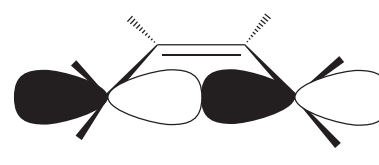

$\sigma^{*}-\mathrm{C}-\mathrm{C}$ orbital
Figure 3: The two orbitals that differentiate each electronic configuration of the CASSCF $(2,2)$ wave function. The HartreeFock configuration is $\Phi_{H F}=\left|\ldots \sigma^{2}\right|$, whereas the other configuration results from the diexcitation, $\sigma \rightarrow \sigma^{*}$, and labeled as charge transfer, $\Phi_{C T}=\left|\ldots \sigma^{* 2}\right|$. The resulting two configurational wave function is $\Psi_{C A S S C F}=C_{H F} \Phi_{H F}+C_{C T} \Phi_{C T} \cdot{ }^{80}$

With the above well known theory on the disrotatory process we have tried to locate the corresponding stationary point using the generalized GAD algorithm. The described CASSCF $(2,2)$ wave function was used for this purpose. We emphasize that the active space is built using the $\sigma$ and $\sigma^{*}$ orbitals of the $C_{3}-C_{4}$ bond of cyclobutene that breaks in the process, see Fig. 3.

We start the search for the $\mathrm{SP}_{2}$ on the PES near the flat $\mathrm{SP}_{1}$ of $\mathrm{C}_{2 v}$ symmetry. The $\mathrm{SP}_{1}$ connects two cis-butadiene minimums resulting by the rotation around the dihedral angle, dihc $=C_{4} C_{1} C_{2} C_{3}$, defined by the carbon skeleton of the molecule. Note that a lot of sideways are possible for a GAD procedure, we are in the 11-dimensional subspace of $\mathrm{C}_{2 v}$ symmetry. Depending on the selected $\mathbf{v}$ and $\mathbf{w}$-vectors the GAD trajectory can lead into a region of the PES of high energy and without a chemical interest. Such unwhished cases are the reason to start the GAD search at a point located approximately in midways between the mentioned $\mathrm{SP}_{1}$ and the searched $\mathrm{SP}$ of index two. From the guessed start point above the mentioned $\mathrm{SP}_{1}$, see Fig. 4, we follow a $\mathrm{GAD}_{2}$ trajectory to the $\mathrm{SP}_{2}$ on the PES. Mainly the dihedrals of the outer $\mathrm{CH}_{2}$ groups are concerned. A picture of two different dihedral coordinates of the run is concurrently shown in Fig. 4, where the distance of the central carbon atoms $\mathrm{C} 1$ and $\mathrm{C} 2$ is drawn against the two dihedrals, $\operatorname{dih} 3=\mathrm{H}_{7} \mathrm{C}_{3} \mathrm{C}_{2} \mathrm{H}_{5}$ and dih6 $=H_{10} C_{4} C_{1} H_{6}$. We had used a step length of the subroutine DOP $853^{58}$ procedure of 0.075 atomic units at the start. This steplength is somewhat stretched by the procedure; but the PES is complicated, and for this reason only small steps lead to the desired results. In the present case the steps should be not larger than 0.2 atomic units. The GAD search finds the region of the $\mathrm{SP}_{2}$. This happens after 667 steps with a result of $|\operatorname{grad}| \leq 10^{-2}$. The further convergence is very slow, see remarks elsewhere. ${ }^{32}$ Comparing with a standard calculation procedure the rule consists in cutting the calculation 'near' the TS of interest, and use, for example, a Newton-Raphson step to quickly reach the TS.

On the right hand side of the center panel of Fig. 4 is reported an SD curve from the starting point of the $\mathrm{GAD}_{2}$ curve. The $\mathrm{SD}$ converges to the non-planar minimum of cis-butadiene. Combining both curves, the $\mathrm{GAD}_{2}$ pathway and the $\mathrm{SD}$, gives a kind of non-RP from the minimum of the cis-butadiene, to the $\mathrm{SP}_{2}$ of the disrotatory process.

To control the disrotatory process we additionally computed the $\mathrm{SP}_{2}$ by GamessUS ${ }^{59,62}$ using $\mathrm{CAS}(4,4)$ selecting as an active space the $\sigma, \sigma^{*}, \pi$ and $\pi^{*}$ as active molecular or- 

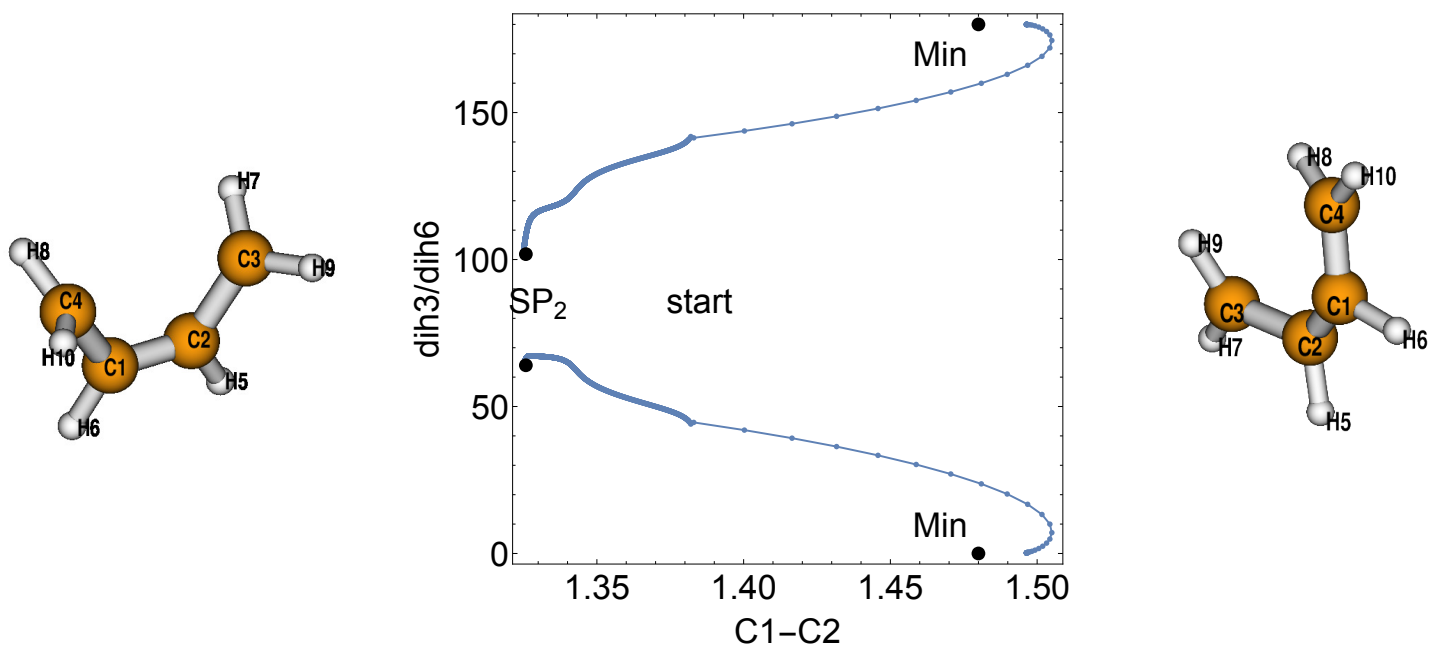

Figure 4: The fat pathway corresponds to the $\mathrm{GAD}_{2}$ curve starting over the planar cisbutadiene isomer (being the $\mathrm{SP}_{1}$ joining the two cis-butadiene conformers), see the structure on the right hand side. It leads to the $\mathrm{SP}_{2}$ related to the disrotatory process (structure on the left hand side). The $\mathrm{GAD}_{2}$ curve is represented by its projection into the plane defined by the two equivalent dihedrals, namely, $\operatorname{dih} 3=H_{7} C_{3} C_{2} H_{5}$ and $\operatorname{dih} 6=H_{10} C_{4} C_{1} H_{6}$, and the skeletal bond distance, $C_{1}-C_{2}$. The numbering is according to Table 1 . The dihedrals hold the symmetry relations, $\operatorname{dih} 3=-\operatorname{dih} 4$ and $\operatorname{dih} 6=-\operatorname{dih} 5$, where $\operatorname{dih} 4=H_{8} C_{4} C_{1} H_{6}$ and $\operatorname{dih} 5=\mathrm{H}_{9} \mathrm{C}_{3} \mathrm{C}_{2} H_{5}$. The right thin curve is an SD curve. See text for more details.

bitals and the four electrons are distributed within this space. The relocalization of the $\mathrm{SP}_{2}$ is carried out by the Schlegel method as implemented in GamessUS. We get the same figures, compare Table 4 . In this way the disrotatory process will be described correctly.

\section{The Topographic Nature of a Two-dimensional Cut of the PES around the Disrotatory $\mathbf{S P}_{2}$}

\section{The SD Exploration}

The discussion of the previous sections on the conrotatory-disrotatory process is well known from not so long ago, see e.g. Ref. 80. The novelty here is the behavior of the GAD concept to find SPs of the non-RP process like the disrotatory ring-opening of the cyclobutene. As noted previously, this well known non-RP evolution of the present isomerization opens the possibility of a 'roaming' mechanism for the reaction. As far as the authors know, this possibility has not been considered yet. The possible existence of a 'roaming' mechanism is strongly related to the nature of the PES around the summit of the $\mathrm{SP}_{2}$ related to the disrotatory motion of the ring opening of cyclobutene. In this section we study in some detail the topography of this region of the PES using geodesic curves like SD. Because the system has a high dimensionality, we focus the topographical study on a slice of the PES that corresponds to a two-dimensional plane characterized by corresponding dihedrals of the outer methylens and the basic $\mathrm{C}_{1}-\mathrm{C}_{2}$ bond distance. The selection is supported by the fact that these molecular geometry parameters are under the strongly changing ones in the reaction. 


\begin{tabular}{rrr}
1.325 & & \\
1.504 & 122.4 & \\
1.479 & 122.8 & 0.0 \\
1.077 & 120.2 & 180.2 \\
1.078 & 119.6 & 179.7 \\
1.073 & 120.4 & 63.2 \\
1.074 & 120.4 & -63.8 \\
1.073 & 120.8 & -105.4 \\
1.074 & 121.8 & 102.5 \\
\hline
\end{tabular}

Table 4: Coordinates of the $\mathrm{SP}_{2}$ - the higher TS in the PES mountains over cyclobutene, ${ }^{80}$ see left structure in Fig. 4. The energy is $-154.8005 \mathrm{au}$. The two decay eigenvalues are here in a relation 5.1 to 3.8 (in au) where the larger one belongs to the disrotatory eigendirection.

We start running an $\mathrm{SD}$ curve from the $\mathrm{SP}_{2}$ along the direction parallel to the eigenvector of the Hessian matrix with a negative eigenvalue and the symmetric eigenvector belonging to the $a_{1}$ irreducible representation. The initial point was taken from $\mathrm{SP}_{2}$ distorted slightly by shortening the two bond angles, $c c c 3=\widehat{C_{1} C_{2} C_{3}}$ and $c c c 4=\widehat{C_{2} C_{1} C_{4}}$, by $-5^{\circ}$. These two bond angles are related by symmetry.

In Figs. 5 and 6 we depict the SD curve projected in the type of planes described above that are defined by dihedrals and the skeletal bond distance. The curve represented by bullets is the $\mathrm{SD}$ that goes from $\mathrm{SP}_{2}$ to the minimum cyclobutene and is embedded in this side of the PES. The symmetry of the dihedrals of the two outer $\mathrm{CH}_{2}$ groups is observed by a comparison of the these two Figures.

Figs. 5 and 6 also contain a second SD curve in a non-symmetric shape depicted as a curve without bullets. Near the $\mathrm{SP}_{2}$ its start point is obtained by addition of the second eigenvector of the Hessian to the summit node, and by distortion of $-13^{\circ}$ in the two $\widehat{C_{1} C_{2} C_{3}}$ and $\widehat{C_{2} C_{1} C_{4}}$ angles.

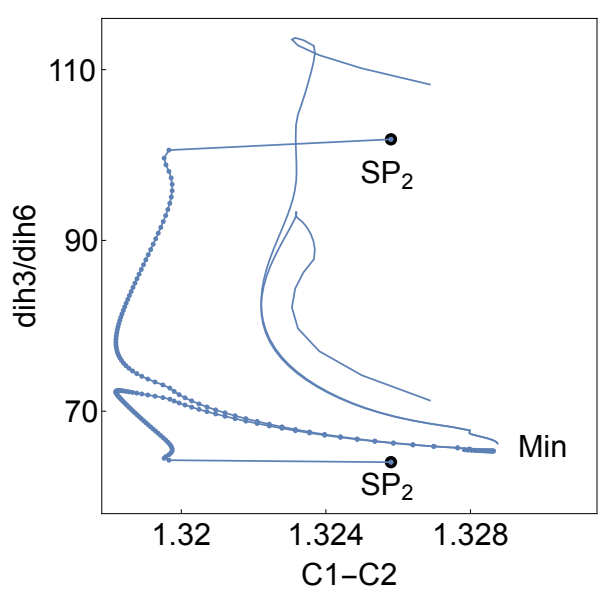

Figure 5: The projection of the SD curve from $\mathrm{SP}_{2}$ to cis-butadiene minimum in the plane defined by the dihedrals, $\operatorname{dih} 3=\mathrm{H}_{7} \mathrm{C}_{3} \mathrm{C}_{2} \mathrm{H}_{5}$ and $\operatorname{dih} 6=H_{10} C_{4} C_{1} H_{6}$ and the skeletal bond distance $C_{1}-C_{2}$. The numbering is from Fig. 1. One curve is depicted with bullets and it initially follows the direction of the eigenpair of negative eigenvalue and symmetric eigenvector. The thin curve is the SD curve that follows the eigenpair of a negative eigenvalue and the antisymmetric eigenvector. It goes to the conrotatory region. See text for more detail.

We remember that this direction is related to the broken symmetry in respect of the symmetry where the $\mathrm{SP}_{2}$ structure belongs. Whereas the first SD curve (with bullets) clearly shows the disrotational symmetry rotational movement of the two outer $\mathrm{CH}_{2}$ groups, the second SD curve shows its symmetric breaking behavior of the original disrotatory symmetry, and thus, its move towards the region of the PES where the conrotatory movement takes place. These results explain the form and character of the surface around the disrotatory $\mathrm{SP}_{2}$ : this point is in the "intersection" of the two ridges. One of these ridges is where the conrotatory $\mathrm{SP}_{1}$ is located and for this reason the set of points of this ridge belongs to the conrotaroty symmetry. The other 


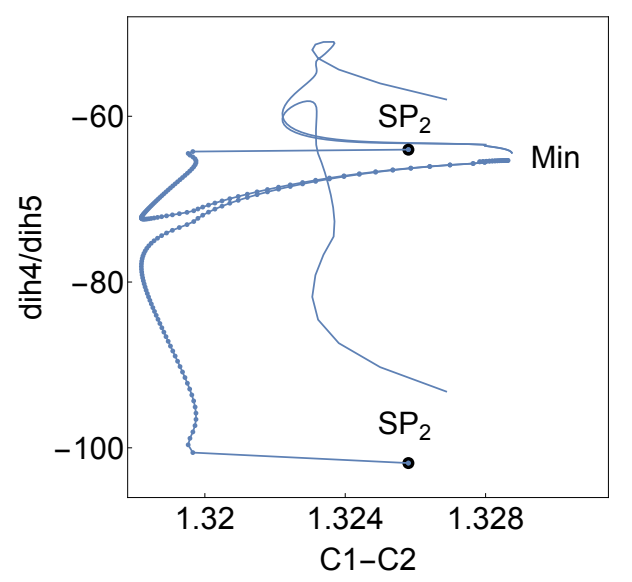

Figure 6: The same as Fig. 5 but using the dihedrals, dih4 $=H_{8} C_{4} C_{1} H_{6}$ and dih5 $=$ $\mathrm{H}_{9} \mathrm{C}_{3} \mathrm{C}_{2} \mathrm{H}_{5}$, for a plane definition.

ridge belongs to the disrotatory symmetry. Additionally, in this second relaxation SD pathway, the two $\mathrm{CH}_{2}$ end groups present a small puckering. There also exist in both SD pathways a confluence channel where the two different dihedrals unite at the same value.

Usually, a small distortion of the $\mathrm{SP}_{2}$ leads to a descent on the other side of the summit to the valley of the PES where the cisbutadiene is located. We report in Fig. 7 a third and a fourth SD curve. The third line represented by the bullets is an expected disrotatory pathway to the nonplanar cisbutadiene minimum. As in the previous case, the SD curve represented by a thin line and initialized with a distortion direction parallel to the antisymmetric eigenvector of the Hessian matrix with negative eigenvalue describes a pathway such that the two outer $\mathrm{CH}_{2}$ groups rotate conrotatorly ending to the cis-butadiene minimum like in the usual conrotatory process.

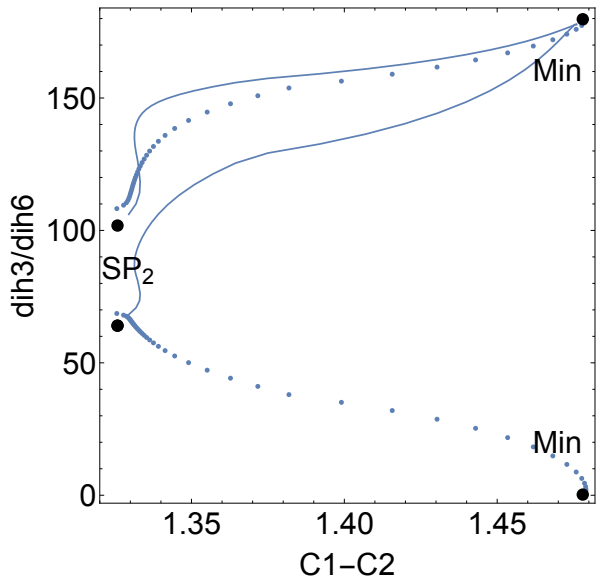

Figure 7: Projection in the plane defined by the dihedrals, $\operatorname{dih} 3=\mathrm{H}_{7} \mathrm{C}_{3} \mathrm{C}_{2} \mathrm{H}_{5}$ and $\operatorname{dih} 6=$ $\mathrm{H}_{10} \mathrm{C}_{4} \mathrm{C}_{1} \mathrm{H}_{6}$, and the bond distance $\mathrm{C}_{1}-\mathrm{C}_{2}$ of two different SD curves joining downhill the $\mathrm{SP}_{2}$ to the nonplanar cis-butadiene minimum. A similar picture emerges if the plane is represented by the dihedrals dih $4=$ $H_{8} C_{4} C_{1} H_{6}$ and $d i h 5=H_{9} C_{3} C_{2} H_{5}$ and the same bond distance.

\section{The NT Exploration: Existence of VRIs Points}

The SD concept does not give information about how and where the potential energy levels change from convex to concave behavior, or vice versa. SD does not describe the curvature of the level hypersurfaces and thus not any bifurcation of a valley. ${ }^{50}$

To achieve this information one has to use the NT concept. ${ }^{51}$ If VRI points exist on a PES they are detected by a singular NT pathway. The usual way to the procedure is to follow any regular NT, and search in a kink region for a VRI. The iteration method to locate VRIs is described in Ref. 57. If a VRI is located then the four branches of the singular NT pathway through the VRI point can be determined. ${ }^{51,83}$ The tangents to the crossing branches at the VRI form a plane in the $\mathbb{R}^{N}$. It is the plane of the bifurcating valleys or ridges of the VRI point. 


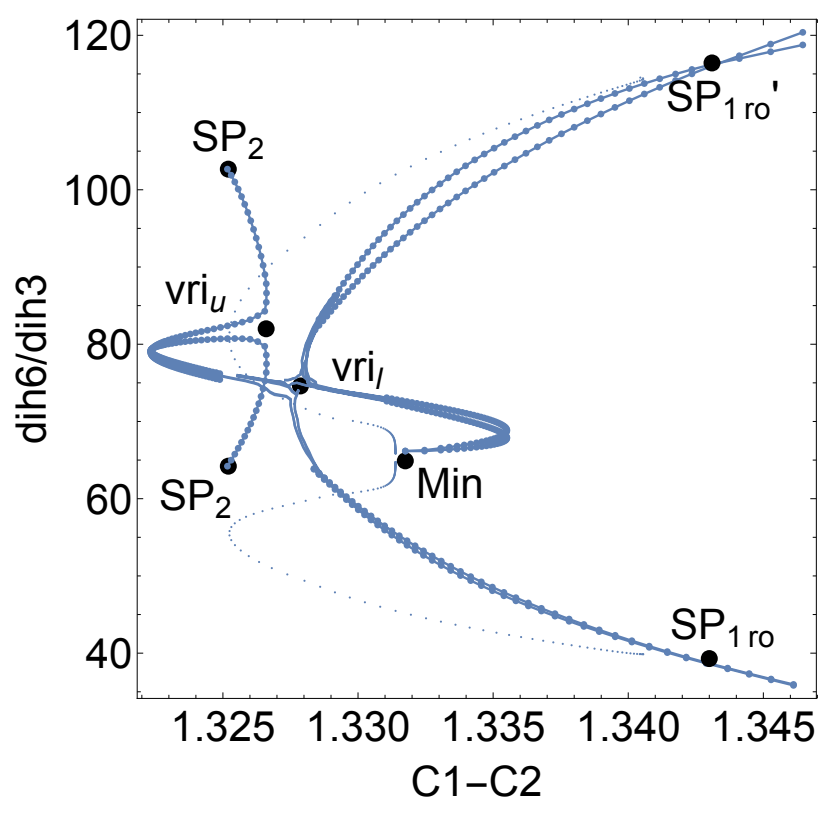

Figure 8: Projection of a singular NT pathway in the plane characterized by the dihedrals, $\operatorname{dih} 3=\mathrm{H}_{7} \mathrm{C}_{3} \mathrm{C}_{2} \mathrm{H}_{5}$ and $\operatorname{dih} 6=$ $H_{10} C_{4} C_{1} H_{6}$, and the bond distance $C_{1}-C_{2}$. The singular NT starts at the cyclobutene minimum (Min), crosses the $\mathrm{VRI}_{l}$, and passes close to the $\mathrm{VRI}_{u}$. Near the $\mathrm{VRI}_{u}$ the NT pathway splits for the two dihedrals and finds the final $\mathrm{SP}_{2}$. Two other branches of the singular NT from $\mathrm{VRI}_{l}$ go to the corresponding $\mathrm{SP}_{1 \text { ro }}$, or $\mathrm{SP}_{1 \text { ro }}$, where 'ro' means ring opening, the transition state of the isomerization of cyclobutene to cis-butadiene. The latter is located on the other side of the mountains, not shown in this figure. The curve of thin points is the steepest descent from $\mathrm{SP}_{1}$ to the minimum.

In the present $\mathrm{PES}$ an important VRI point is the one which is reported in Fig. 8 (and in Table 5). This "lower" VRI, hereafter labeled as $\mathrm{VRI}_{l}$, is a candidate for the "global" VRI if one starts the NT pathway from the cyclobutene minimum. From the energy point of view, the VRI point is located in between the $\mathrm{SP}_{1}$ and $\mathrm{SP}_{2}$. Starting from the cyclobutene minimum, the NT pathway locates this VRI. In more detail, the NT path first follows a valley from the cyclobutene minimum uphill ending at the $\mathrm{VRI}_{l}$ and from this point tree other branches go further along

\begin{tabular}{rrr}
\hline 1.326 & & \\
1.480 & 109.9 & \\
1.501 & 108.5 & -1.2 \\
1.077 & 126.2 & -179.9 \\
1.076 & 127.1 & 180.0 \\
1.078 & 119.4 & 76.1 \\
1.076 & 119.4 & -74.1 \\
1.090 & 119.9 & -74.1 \\
1.077 & 119.1 & 76.0 \\
\hline
\end{tabular}

Table 5: Coordinates of a VRI point in the PES mountains over cyclobutene, see Fig. 8. It is named $\mathrm{VRI}_{l}$. Its energy is $-154.8171 \mathrm{au}$.

ridges. Due to this topography around the region where the $\mathrm{VRI}_{l}$ is located we say that the PES shows a ridge-bifurcation at this point. ${ }^{50}$ (The bullets are calculated points of the NT pathway. Near the $\mathrm{VRI}_{l}$ the bullets are kept off for visibility.)

\begin{tabular}{rrr}
\hline 1.328 & & \\
1.478 & 127.2 & \\
1.483 & 127.1 & 0.1 \\
1.081 & 117.3 & -178.8 \\
1.080 & 117.5 & 179.1 \\
1.083 & 120.6 & 84.4 \\
1.074 & 120.4 & -81.1 \\
1.068 & 120.5 & -83.6 \\
1.075 & 120.2 & 82.1 \\
\hline
\end{tabular}

Table 6: Coordinates of the $\mathrm{VRI}_{u}$, see Figs. 8 and 10. Its energy is $-154.7986 \mathrm{au}$.

All the NTs that start at the cyclobutene minimum and which do not pass through the $\mathrm{VRI}_{l}$, are called regular NTs. They follow a conrotatory turning of the outer methylenes during the evolution of the curve. So to say, in the $\mathrm{VRI}_{l}$ the conrotatory region of the PES, or more specifically, the region with $\mathrm{C}_{2}$ symmetry characterizing the conrotatory process ends. Energetically, above this $\mathrm{VRI}_{l}$ starts the region of the additional disrotatory process, being throughout of $\mathrm{C}_{s}$ symme- 
try, the symmetry related to the disrotatory movement.

From this view, the $\mathrm{VRI}_{l}$ represents a transition between the $\mathrm{C}_{2}$ symmetry and the $\mathrm{C}_{s}$ symmetry regions of the PES and for this fact the molecular structure of the $\mathrm{VRI}_{l}$ point belongs to the $\mathrm{C}_{1}$ symmetry. In Fig. 8 we show the singular NT going from the cyclobutene minimum to the node labeled $\mathrm{VRI}_{l}$. In this Figure the singular NT curve is projected in the plane characterized by both dihedrals, dih $3=H_{7} C_{3} C_{2} H_{5}$ and $\operatorname{dih} 6=\mathrm{H}_{10} \mathrm{C}_{4} \mathrm{C}_{1} \mathrm{H}_{6}$, and the bond distance $C_{1}-C_{2}$. Note that we present the curves of two dihedral coordinates in one picture. The NT curve leaves the $\mathrm{VRI}_{l}$ node and leads up to a new VRI point located in the disrotatory region of the PES. This new VRI is labeled $\mathrm{VRI}_{u}$ like 'up'. The NT does not cross the new VRI, it touches the region. We can say that the NT is singular in respect to the $\mathrm{VRI}_{l}$ but behaves regular in respect of the $\mathrm{VRI}_{u}$. Near the $\mathrm{VRI}_{u}$ the NT pathway splits in the two dihedrals and finds its final $\mathrm{SP}$, namely, $\mathrm{SP}_{2}$ related to the disrotatory process. Finally, each one of the other two branches of the singular NT from $\mathrm{VRI}_{l}$ ends at the corresponding $\mathrm{SP}_{1 \text { ro }}$, the transition state of the conrotatory isomerization of cyclobutene to cis-butadiene.

This singular NT joining the cyclobutene minimum and the $\mathrm{SP}_{1}$ through the $\mathrm{VRI}_{l}$ is not a usual RP. In fact there is a shorter geodesic path on the $\mathrm{PES}^{20,84}$ along a valley that joins these two stationary points, which is the SD curve being a true RP. The singular $\mathrm{NT}$ is the roundabout way from cyclobutene over the $\mathrm{VRI}_{l}$ to the conrotatory first order saddle point, $\mathrm{SP}_{1}$. The $\mathrm{VRI}_{l}$ can be seen as a non-chemical kink in the pathway, see Fig. 8. The singular NT is the last border for direct conrotatory trajectories. The other singular branch of the NT uphill connects the $\mathrm{VRI}_{l}$ with the $\mathrm{SP}_{2}$ which is related to the disrotatory evolution.

The sub-arc of the NT between $\mathrm{VRI}_{l}$ and $\mathrm{VRI}_{u}$ is located on a ridge, but at the $\mathrm{VRI}_{u}$ does not emerge a new valley. There the ridge bifurcates in three ridges of index two. Two of the branches from $\mathrm{VRI}_{u}$ go slowly downhill in their potential energy to each one of the two symmetric $\mathrm{SP}_{2}$. The $\mathrm{VRI}_{u}$ shows a ridge-bifurcation form of a higher index. In Fig. 8 we show the behavior of the two NTs after its departure from $\mathrm{VRI}_{u}$, the further uphill branch to higher energies is not shown. However this NT arrives to a point of a seam characterized by the intersection of the PESs associated with the singlet and triplet electronic states. At this point the energy difference, $\mathrm{E}$ (Triplet) $-\mathrm{E}($ Singlet $)=1.1 \mathrm{kcal} / \mathrm{mol}$. The $\mathrm{VRI}_{u}$ is near this seam. The lower PES is not smooth in this region.

We note that this is an example showing that the NT concept can be used to detect regions of intersection between electronic states. ${ }^{85}$ In addition we say that the finding is consistent with previous theoretical studies on the isomerization cyclobutenecis-butadiene, that is, a photoexcited cyclobutene follows a disrotatory ring opening. ${ }^{86}$ The $\mathrm{VRI}_{u}$ is located in the disrotatory region of the PES.

It is interesting to observe that the 2dimensional bifurcation planes of the two VRI points, namely, $\mathrm{VRI}_{l}$ and $\mathrm{VRI}_{u}$, of Fig. 8 are quite different planes in the full coordinate space. The $\mathrm{VRI}_{l}$ gives the direction for the conrotatory process whereas the $\mathrm{VRI}_{u}$ for the disrotatory process.

We remark that the reported VRIs are usually 'only' representatives of so-called VRI-manifolds. ${ }^{87,88}$ Other VRI points emerge nearby if other coordinates something change which are not directly involved into the VRI bifurcation: then the dihedrals $\left(\operatorname{dih} 3=H_{7} C_{3} C_{2} H_{5}\right) /\left(\operatorname{dih} 6=H_{10} C_{4} C_{1} H_{6}\right)$ or $\left(\operatorname{dih} 4=H_{8} C_{4} C_{1} H_{6}\right) /\left(\operatorname{dih} 5=H_{9} C_{3} C_{2} H_{5}\right)$ 


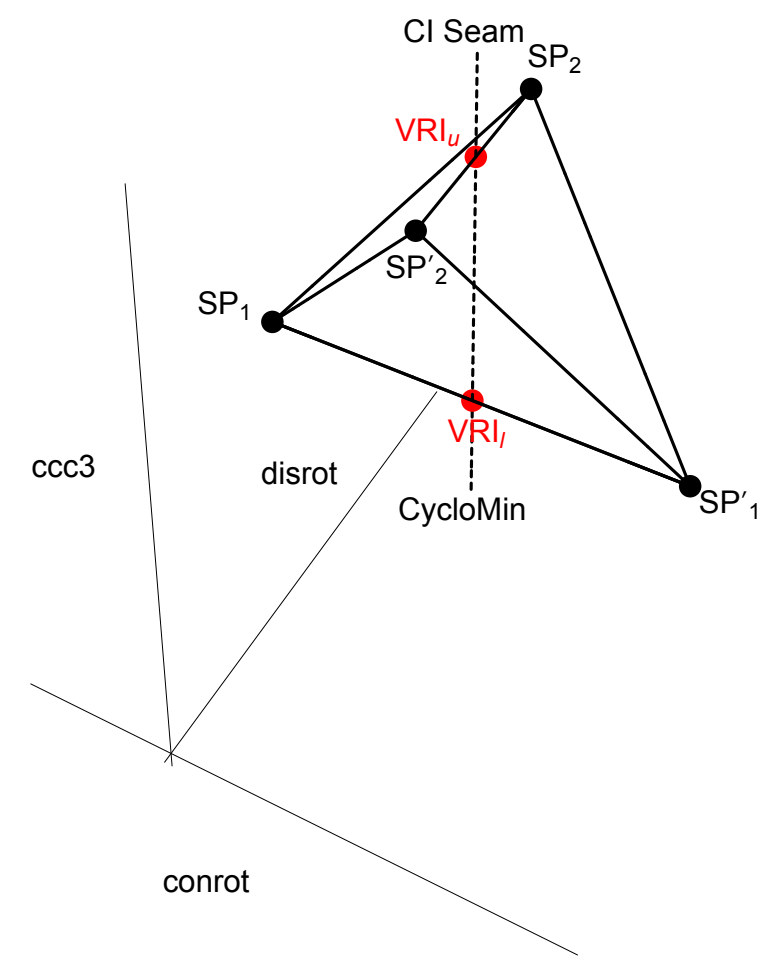

Figure 9: Schematic relation of the different SPs and two VRI points corresponding for the disrotatory against the conrotatory behavior of the dihedrals. The axes 'conrot', as well as 'disrot' depict subspaces of the 24dimensional coordinate space. The dashed central axis of the tetrahedron represents a region in the fully symmetric subspace. The singular NT of Fig. 8 here is represented by parts of the vertical axis and the horizontal edges. See text for a detailed explanation.

show again the typical pattern of a VRI. Often there is a larger-dimensional manifold of VRI points, and our 1-dimensional search method $^{57}$ cannot so easily decide which VRI in which sub-plan should be searched.

To view all previous findings we present a basic general scheme. It emphasizes the relation 'point - opening angle - conrotatory/disrotatory direction', see Fig. 9. In the scheme the different directions of the involved NT-branches for the disrotatory against the conrotatory opening of the molecule are given explicitly. Note that every axis means a subspace of the corresponding coordinates. To depict Fig. 9, we need the representation in a three-dimensional tetrahedron scheme to draw all possible connections in this symmetry version. It would not be possible to understand the behavior of the PES in a planar representation. In Fig. 9, every symmetry version of the $\mathrm{SP}_{2}$ can be connected by NTs with every symmetry version of the $\mathrm{SP}_{1}$, the transition state of the conrotatory process. All diverse special nodes involved in the scheme are assumed to be of the symmetry-version

$\operatorname{dih} 3+\operatorname{dih} 4-\operatorname{dih} 5-\operatorname{dih} 6+$.

Sometimes also the dihedral angle dihc $=$ $C_{4} C_{1} C_{2} C_{3}$ can jump from plus to minus, for the $\mathrm{SP}_{1}$ of the ring opening, for example. That is an extra symmetry, which is not included in the scheme, especially because most nodes of interest have dihc $\approx 0$.

\begin{tabular}{rrrrr} 
& $\mathrm{SP}_{2} \rightarrow \mathrm{SP}_{1 r o}$ & \multicolumn{2}{c}{$\mathrm{SP}_{2} \rightarrow \mathrm{SP}_{1 r o}$ ' } \\
\hline dih3 & $102 \rightarrow$ & 38 & $102 \rightarrow$ & 116 \\
dih4 & $-102 \rightarrow$ & -116 & $-102 \rightarrow$ & -38 \\
dih5 & $-64 \rightarrow$ & -116 & $-64 \rightarrow$ & -38 \\
dih6 & $64 \rightarrow$ & 38 & $64 \rightarrow$ & 116 \\
\hline
\end{tabular}

\begin{tabular}{rrrrr} 
& \multicolumn{2}{c}{$\mathrm{SP}_{2} \prime \rightarrow \mathrm{SP}_{1 r o}$} & \multicolumn{2}{c}{$\mathrm{SP}_{2} \prime \rightarrow \mathrm{SP}_{1 \text { ro }} \prime$} \\
\hline dih3 & $64 \rightarrow$ & 38 & $64 \rightarrow$ & 116 \\
dih4 & $-64 \rightarrow-116$ & $-64 \rightarrow$ & -38 \\
dih5 $-102 \rightarrow$ & -116 & $-102 \rightarrow$ & -38 \\
dih6 & $102 \rightarrow$ & 38 & $102 \rightarrow$ & 116 \\
\hline
\end{tabular}

Table 7: Four regular NTs joining SPs of index-2 $\left(\mathrm{SP}_{2}\right)$ to SPs of index-1 $\left(\mathrm{SP}_{1}\right)$, all are located on the barrier ridge of the ring opening (ro) of cyclobutene.

The tetrahedron represents:

(i) The skew edges of the tetrahedron connect SPs of a different index. They are 
regular NTs. These NTs in the scheme are understood by the connections given in Table 7. Additionally, the scheme is a nice example of the index theorem for NTs. ${ }^{83}$

(ii) The upper edge is the disrot (disrotatory) subspace of the dihedrals dih $3=$ $H_{7} C_{3} C_{2} H_{5}$, dih $4=H_{8} C_{4} C_{1} H_{6}$, dih $5=$ $\mathrm{H}_{9} \mathrm{C}_{3} \mathrm{C}_{2} \mathrm{H}_{5}$ and $\operatorname{dih} 6=\mathrm{H}_{10} \mathrm{C}_{4} \mathrm{C}_{1} \mathrm{H}_{6}$, and

(iii) the lower edge is the conrot (conrotatory) subspace of the dihedrals dih $3=$ $\mathrm{H}_{7} \mathrm{C}_{3} \mathrm{C}_{2} \mathrm{H}_{5}$, dih $4=\mathrm{H}_{8} \mathrm{C}_{4} \mathrm{C}_{1} \mathrm{H}_{6}$, dih $5=$ $\mathrm{H}_{9} \mathrm{C}_{3} \mathrm{C}_{2} \mathrm{H}_{5}$ and dih6 $=\mathrm{H}_{10} \mathrm{C}_{4} \mathrm{C}_{1} \mathrm{H}_{6}$. Both edges are somehow orthogonal.

(iv) The vertical axis is approximately in the totally symmetric subspace of the molecule.

In Fig. 9 the vertical direction is the ringopening angle, but it can also be the energy. Located on or near the vertical axis are the $\mathrm{VRI}_{l}$ and $\mathrm{VRI}_{u}$ points.

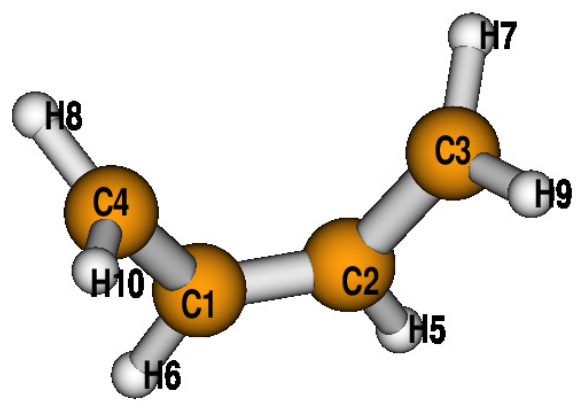

Figure 10: Structure of cyclobutenes $\mathrm{VRI}_{u}$ point, see Table 6 and Fig. 8.

From the $\mathrm{VRI}_{l}$ point the singular $\mathrm{NT}$ evolves to the two $\mathrm{SP}_{1}$ and $\mathrm{SP}_{1}$ ' near the disrotatory edge, from the $\mathrm{VRI}_{u}$ point the $\mathrm{NT}$ evolves further to the two $\mathrm{SP}_{2}$ and $\mathrm{SP}_{2}$ ' near the conrotatory edge, and the four other skew edges of the tetrahedron are the schematic NTs connecting the two SPs of index-2 $\left(\mathrm{SP}_{2}\right)$ and the two SPs of index-1 $\left(\mathrm{SP}_{1}\right)$. Finally, the cyclobutene minimum is located below, but at the top of the central line is the seam due to the crossing between the singlet-triplet electronic states, which is reported above.

In an older treatment ${ }^{89}$ one considered the symmetry of the whole process (conrotatory and disrotatory) and a "ball as a representation of the system PES". In the two poles of the ball the two $\mathrm{SP}_{2}$ associated with the disrotarory process are located. Note that in the disrotatory process one $\mathrm{SP}$ is a mirror image of the other. In a latitude over the equatorial plane the two $\mathrm{SP}_{1}$ of the conrotatory process are located. These two $\mathrm{SP}_{1}$ are the images of the two $\mathrm{SP}_{1}$ located in the other latitude. The ball has a mirror symmetry through the equator and the mirror is characterized by the diameter being perpendicular to the equatorial plane which passes through the poles where the above two $\mathrm{SP}_{2}$ are located. In this representation two symmetric $\mathrm{SP}_{2}$ and four symmetric $\mathrm{SP}_{1}$ (with \pm dihc $= \pm C_{4} C_{1} C_{2} C_{3}$ ) plus the corresponding VRIs are included.

\section{Further Regions of the PES Explored by NTs Starting from $\mathrm{SP}_{2}$}

In the last section we have explored the regions of the PES where the cyclobutene minimum, the conrotatory $\mathrm{SP}_{1}$ and the disrotatory $\mathrm{SP}_{2}$ are located by the NT concept. Now using the same concept we expect to explore the other side of the PES, the butadiene bowl. Starting from $\mathrm{SP}_{2}$, we found NTs which arrive at another $\mathrm{SP}_{1}$ on the cis-butadiene side. It is an $\mathrm{SP}_{1}$ with one $\mathrm{CH}_{2}$ group in plane, the other nearly $90^{\circ}$ turned, for this reason it is labeled as $\mathrm{SP}_{1 \text { orthogonal }}=\mathrm{SP}_{1 \perp}$. The molecular geometry of the $\mathrm{SP}_{1 \perp}$ is given in Table 8 and Fig. 11. 


\begin{tabular}{rrr}
\hline 1.340 & & \\
1.476 & 125.8 & \\
1.436 & 125.9 & 0.0 \\
1.078 & 117.9 & 180.0 \\
1.076 & 117.7 & 180.0 \\
1.074 & 120.8 & 83.8 \\
1.071 & 121.6 & -180.0 \\
1.075 & 120.7 & -83.8 \\
1.093 & 120.6 & 0.0 \\
\hline
\end{tabular}

Table 8: The molecular geometry of an $\mathrm{SP}_{1 \perp}$. This SP is located in the cis-butadiene bowl. The energy is $-154.8122 \mathrm{au}$.

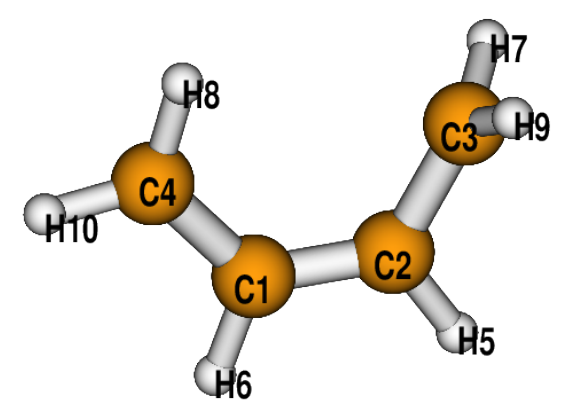

Figure 11: $\mathrm{SP}_{1}$ " orthogonal", see Table 8, in the cis-butadiene well.

Other symmetry versions can emerge here where the two outer $\mathrm{CH}_{2}$ groups are interchanged. Analogously to the tetrahedron scheme of Fig. 9, we have similar symmetries in the present case, see Table 9.

From the summit of the $\mathrm{SP}_{2}$ we can follow different trajectories which find different ridges between the valleys in the cisbutadiene bowl. The NT concept is well adapted for such explorations. These NTs find a "second" channel which the $\mathrm{SP}_{2}$ opens, besides the conrotatory channel along one of the eigenvectors in analogy to the usual $\mathrm{SP}_{1}$. But it is still not the transition to the transbutadiene, the global minimum.

In Fig. 12 a regular NT is shown which connects the $\mathrm{SP}_{1 \perp}$ with the other stationary points reported already in this work, on the

\begin{tabular}{|c|c|c|c|}
\hline \multicolumn{2}{|r|}{$\mathrm{SP}_{2} \rightarrow \mathrm{SP}_{1 \perp}$} & \multicolumn{2}{|c|}{$\mathrm{SP}_{2} \rightarrow \mathrm{SP}_{1 \perp}$} \\
\hline $\operatorname{dih} 3$ & $102 \rightarrow \quad 84$ & $102 \rightarrow$ & 0 \\
\hline $\operatorname{dih} 4$ & $-102 \rightarrow-180$ & $-102 \rightarrow$ & -83 \\
\hline $\operatorname{dih} 5$ & $-64 \rightarrow \quad-83$ & $-64 \rightarrow$ & -180 \\
\hline $\operatorname{dih} 6$ & $64 \rightarrow$ & $64 \rightarrow$ & 84 \\
\hline \multicolumn{2}{|r|}{$\mathrm{SP}_{2} \prime \rightarrow \mathrm{SP}_{1 \perp}$} & \multicolumn{2}{|c|}{$\mathrm{SP}_{2} \prime \rightarrow \mathrm{SP}_{1 \perp}^{\prime}$} \\
\hline $\operatorname{dih} 3$ & $64 \rightarrow \quad 84$ & $64 \rightarrow$ & 0 \\
\hline $\operatorname{dih} 4$ & $-64 \rightarrow-180$ & $-64 \rightarrow$ & -83 \\
\hline $\operatorname{dih} 5$ & $-102 \rightarrow \quad-83$ & $-102 \rightarrow$ & -180 \\
\hline $\operatorname{dih} 6$ & $102 \rightarrow$ & $102 \rightarrow$ & 84 \\
\hline
\end{tabular}

Table 9: Four regular NTs from $\mathrm{SP}_{2}$ to $\mathrm{SP}_{1} \perp$ on the cis-butadiene side of the 2-dimensional barrier.

ridge, and in the cyclobutene bowl. Here we use for the second coordinate one of the two ring opening angles, $c c c 3=\widehat{C_{1} C_{2} C_{3}}$. The wideness of the space of the two NTs between the cyclobutene minimum and the $\mathrm{SP}_{1}$ of the ring opening may give an impression of the reaction channel ${ }^{83}$ which may be defined by the full family of all regular NTs between the two stationary nodes.

In Fig. 13 we additionally represent the energy profile for the NT of Fig. 12. Note that in the regions where the NT climbs quite highly into the mountains of the PES, the curve has two turning points (TP). Shortly behind these TPs begins a region where a seam is located due to the crossing between the singlet and the triplet electronic state surfaces.

\section{VRI point in the cis-butadiene well}

In the cis-well of the butadiene side, after the $\mathrm{SP}_{2}$, there exist two saddles of index one, the $\mathrm{SP}_{1 \text { ro }}$ and the $\mathrm{SP}_{1 \perp}$, compare Fig. 12. Following the index theorem for NTs, a VRI point has to emerge between them. The singular NT which belongs to the VRI point should connect the two SPs of index one, as 


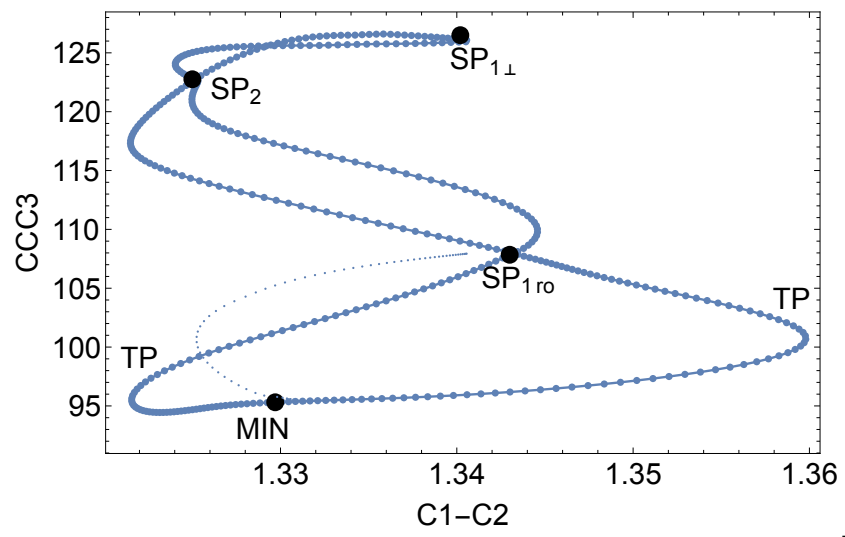

Figure 12: An example of a regular NT evolving from $\mathrm{SP}_{1 \perp}$, backward uphill to the $\mathrm{SP}_{2}$ of the disrotatory ring opening. From this point it goes downhill to the usual $\mathrm{SP}_{1 \text { ro }}$ of the conrotatory ring opening, and it goes further over a turning point (TP) and finally down to the cyclobutene minimum (MIN). There the dihedral dihc $=C_{4} C_{1} C_{2} C_{3}$ changes the sign, and another NT goes back through all previous stationary points to $\mathrm{SP}_{1 \perp}$. The $\mathrm{NT}$ is projected in the plane characterized by the bond angle $\widehat{C_{1} C_{2} C_{3}}$ and the bond distance $C_{1}-C_{2}$. The curve with thin points is the $\mathrm{SD}$ from $\mathrm{SP}_{1 \text { ro }}$. Note that the butadiene minimums are 'far away' behind the right hand side of the picture.

well as the $\mathrm{SP}_{2}$. We report in Fig. 14 such a $\mathrm{VRI}_{\text {cis }}$ point. Three branches fullfil the connections above, and the forth branch goes for an increasing $C_{1}-C_{2}$-distance quickly into a region with a seam intersection. The "left" branch from $\mathrm{SP}_{2}$ to the VRI is a ridge of index two, the two branches from the VRI point to the two SPs of index one are ridges of index one, as well as the horizontal branch to the right hand side. So to say, the region of the disrotatory $\mathrm{SP}_{2}$ with two decay directions ends at the VRI point.

Note that the $\mathrm{VRI}_{\text {cis }}$ point is a representative of a higher dimensional VRI manifold. ${ }^{87,88}$ The $\mathrm{SP}_{2}$ is connected with the $\mathrm{SP}_{1 \perp}$

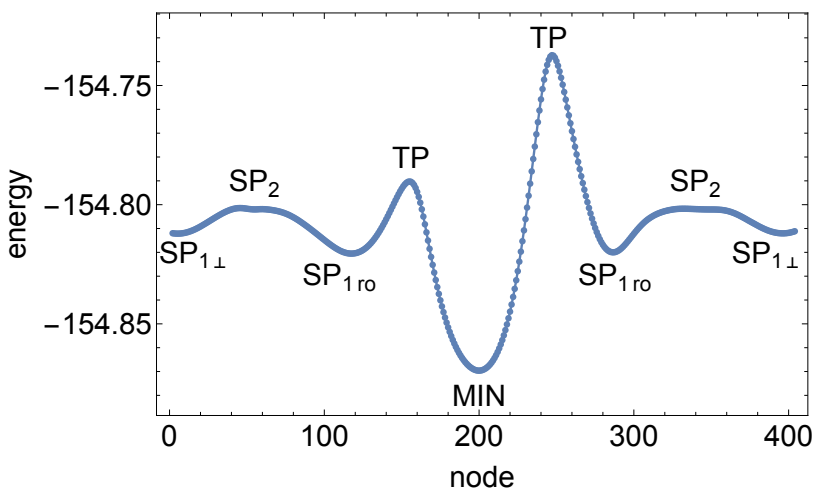

Figure 13: Energy profile (in $a u$ ) over the regular NT represented in Fig. 12. The explanation of the symbols is given in Fig. 12.

by infinitely many regular NTs, as well as it is connected with the $\mathrm{SP}_{1 \text { ro }}$ by infinitely many regular NTs. However between the two saddles of index one only the singular NT exists; for every constellation of the other coordinates maximal one singular NT exists which crosses one VRI point.

\section{The Transformation: cis- Butadiene to trans-Butadiene}

The isomerization of the cyclobutene just discussed leads to cis-butadiene, however, this compound is transformed by a simple rotation around the bond distance $C_{1}-C_{2}$ to the most stable conformational isomer, namely, the flat trans-butadiene. In this subsection we study the region of the PES associated with this conformational transformation for completeness and also as a test for the GAD concept. The GAD algorithm searches an RP on the very flat butadiene side of the PES. Starting at the planar structure of cis-butadiene, the GAD method based in the Eqs. 9 and 10 locates the cis-butadiene minimum, and an $\mathrm{SP}_{1}$ associated with the rotation of the dihedral angle to dihc $=$ $C_{4} C_{1} C_{2} C_{3}=101.4^{\circ}$. The $\mathrm{SP}_{1}$ is already reported in Ref. 90. Turning further the di- 


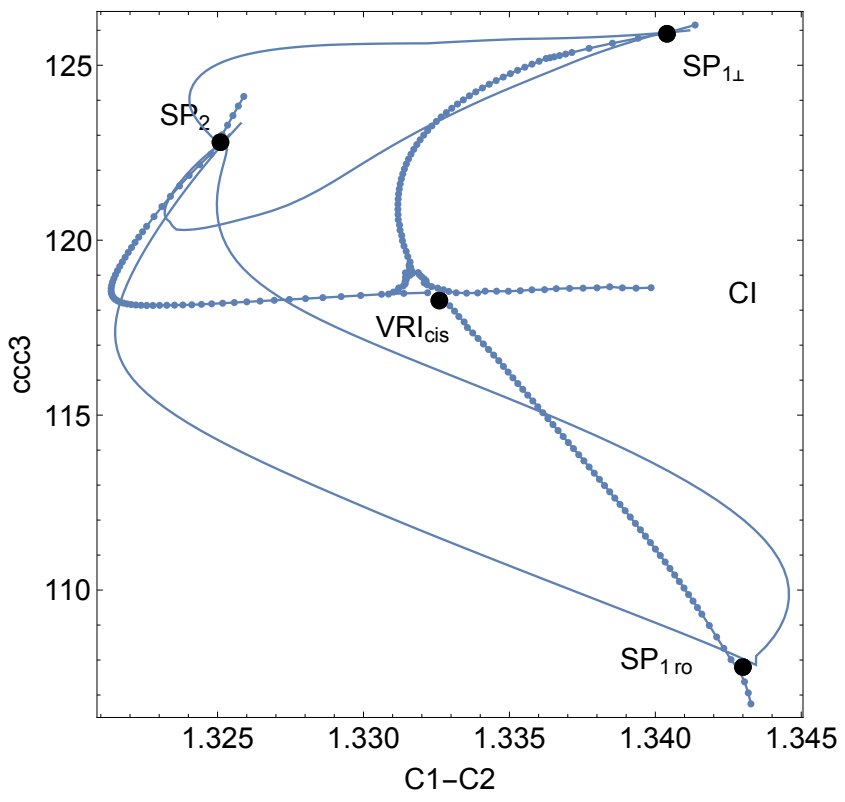

Figure 14: Projection of the singular NT (curve with bullets) connecting $\mathrm{SP}_{1 \perp}, \mathrm{SP}_{2}$ and $\mathrm{SP}_{1 \text { ro }}$ via a VRI point, named $\mathrm{VRI}_{\text {cis }}$. The NT is projected in the plane characterized by the bond angle $\widehat{C_{1} C_{2} C_{3}}$ and the bond distance $C_{1}-C_{2}$. Some regular NTs (thin lines) are also included. (Note: there is no regular $\mathrm{NT}$ between $\mathrm{SP}_{1 \perp}$ and $\mathrm{SP}_{1 \text { ro }}$.)

hedral angle, dihc, gives the planar transbutadiene minimum.

\section{Discussion and Conclusions}

We have shown the applicability of the GAD method to a further example of a small molecule, see other examples elsewhere. ${ }^{22,31,32}$ We use the coordinate systems of internal coordinates where the curvilinear metric has to be included in the GAD formulas. The GAD method is a 'growing string' method for the exploration of a PES like following an NT. Both methods often find the region of interest of the SP. GAD has two problematic aspects: First, the sticking point is the handicap of the initial point and the initial help vectors. Thus, one has to guess an abstract region of attractivity of the corresponding SP (which is, of course, usually unknown). In Ref. 24 the authors worked with an 'available good initial guess': a coarse minimum energy path is determined before by a double-ended string method. The second drawback is a very slow convergence of the DOP853 procedure in the neighborhood of a found stationary point. To exactly localize the SP is usually not very effective, compare also Ref. 26. This can be solved under observation of the norm of the gradient of the current point: if the norm is sufficiently small, one can use another method.

Probably the GAD algorithm is good for chemical systems with metals rather than for flexible organic molecules with a rugged PES.

The NT concept analogously needs a search direction at the beginning. However, the full coordinate space is divided by the singular NTs which delimit regions of attraction of the corresponding SPs of interest. If one can calculate the corresponding VRI points ${ }^{57}$ then one has such a partition, see Fig. 8. The property makes NTs somewhat more interesting than GAD trajectories.

The potentiality of both methods, GAD or NT, to explore PESs has been demonstrated.

From a chemical point of view, the existence of pathways of the ring opening for cyclobutene to quite different regions, down from $\mathrm{SP}_{2}$, is important.

The usual name 'disrotatory' $\mathrm{SP}_{2}$ obscures the fact that the character of the $\mathrm{SP}$ is twofold. It is a two-dimensional barrier. One col direction is conrotatory, but the other one is disrotatory. The $\mathrm{SP}_{1}$ of the 'conrotatory' ring opening is just a pinpoint on the full conrotatory ridge which goes up to the $\mathrm{SP}_{2}$. However, the disrotatory process is high in energy because starting from cyclobutene, the $C_{3}-C_{4} \sigma$-bond is broken and 


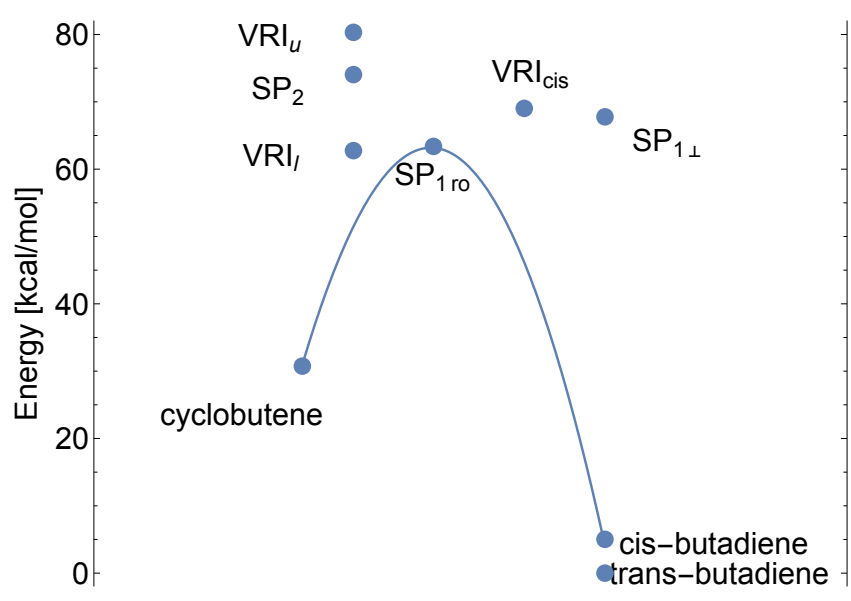

Figure 15: Schematic representation of the energies of some nodes of the ring opening of cyclobutene to butadiene.

the new two $\pi$ bonds are not made until the molecule crosses the $\mathrm{SP}_{2}$ ridge. Even more the system decreases in energy after the $\mathrm{SP}_{2}$ by increasing the distance between the broken $C_{3}-C_{4}$ bond. But still the two $\pi$ bonds are not formed until the ridge of the planar $\mathrm{SP}_{1}$ with $\mathrm{C}_{2 v}$ symmetry is found. (This $\mathrm{SP}_{1}$ with dihc $=C_{4} C_{1} C_{2} C_{3}=0^{\circ}$ is between the two symmetric cis-butadiene minima with $\operatorname{dihc}= \pm 36.4^{\circ}$.)

Due to the formation of these two bonds the energy decreases until the cis-butadiene is formed. Note that along the pure conrotatory process of the ring opening the reaction path is $\mathrm{C}_{2}$ so the bond breaking and bond formation occurs almost simultaneously and for this reason this process is lower in energy.

The reasoning is based on the so-called Bell-Evans-Polanyi (BEP) concept. ${ }^{91-93}$ For the understanding and for rationalizing the $\mathrm{BEP}$ concept is better here than the restrictive Woodward-Hoffmann rules, ${ }^{71,72}$ because no symmetry restrictions are imposed. In fact its extension due to Shaik ${ }^{94}$ offers an easy way to rationalize the concept of forbidden and allowed reactions. An important conclusion of the present study is that from the exploration of the topography of the PES around the $\mathrm{SP}_{2}$ region using the NT concept this region may favour a possible existence of a roaming mechanism. Dynamic studies are necessary to prove this statement. If this fact is confirmed then the NT concept can be used to predict the existence of roaming mechanisms rather than to use expensive dynamic calculations.

For comparison, the energy differences of the reported nodes of this paper are schematically shown in Fig. 15. Note that all these calculations have been obtained using the $\operatorname{CASSCF}(2,2)$ functional as explained above. The arc in Fig. 15 should symbolize the usual $\mathrm{RP}$ between cyclobutene, $\mathrm{SP}_{1 \text { ro }}$ and cisbutadiene. See also the Cartoon in Fig. 16. If the cyclobutene molecule has a little more energy than the barrier of the $\mathrm{SP}_{1 \text { ro }}$, then it can surmount the ridge from $\mathrm{SP}_{1 \text { ro }}$ to $\mathrm{SP}_{2}$ in a conrotatory direction. With still higher energy, the crossing of the ridge anywhere changes to a disrotatory direction, as it is the case also at the top of the $\mathrm{SP}_{2}$.

There is a deeper problem on the cyclobutene disrotatory ring opening. The highest energy point of the disrotatory path has $\mathrm{C}_{s}$ symmetry with two negative eigenvalues. One of the eigenvectors belong to the irreductible representation labeled as a'. This eigenvector is related to the disrotarory evolution. The other eigenvector belongs to the irreductible representation labeled as a". This eigenvector is related to the broken symmetry evolution, however this evolution should arrive at stationary points of symmetry $\mathrm{C}_{2 v}$ (cyclobutene) and $\mathrm{C}_{2}$ (butadiene). Now, $\mathrm{C}_{s}$ is a subset of the $\mathrm{C}_{2 v}$ symmetry group, but $\mathrm{C}_{s}$ is not a subset of the $\mathrm{C}_{2}$ symmetry group. In other words, if we start the calculation at a point of the 


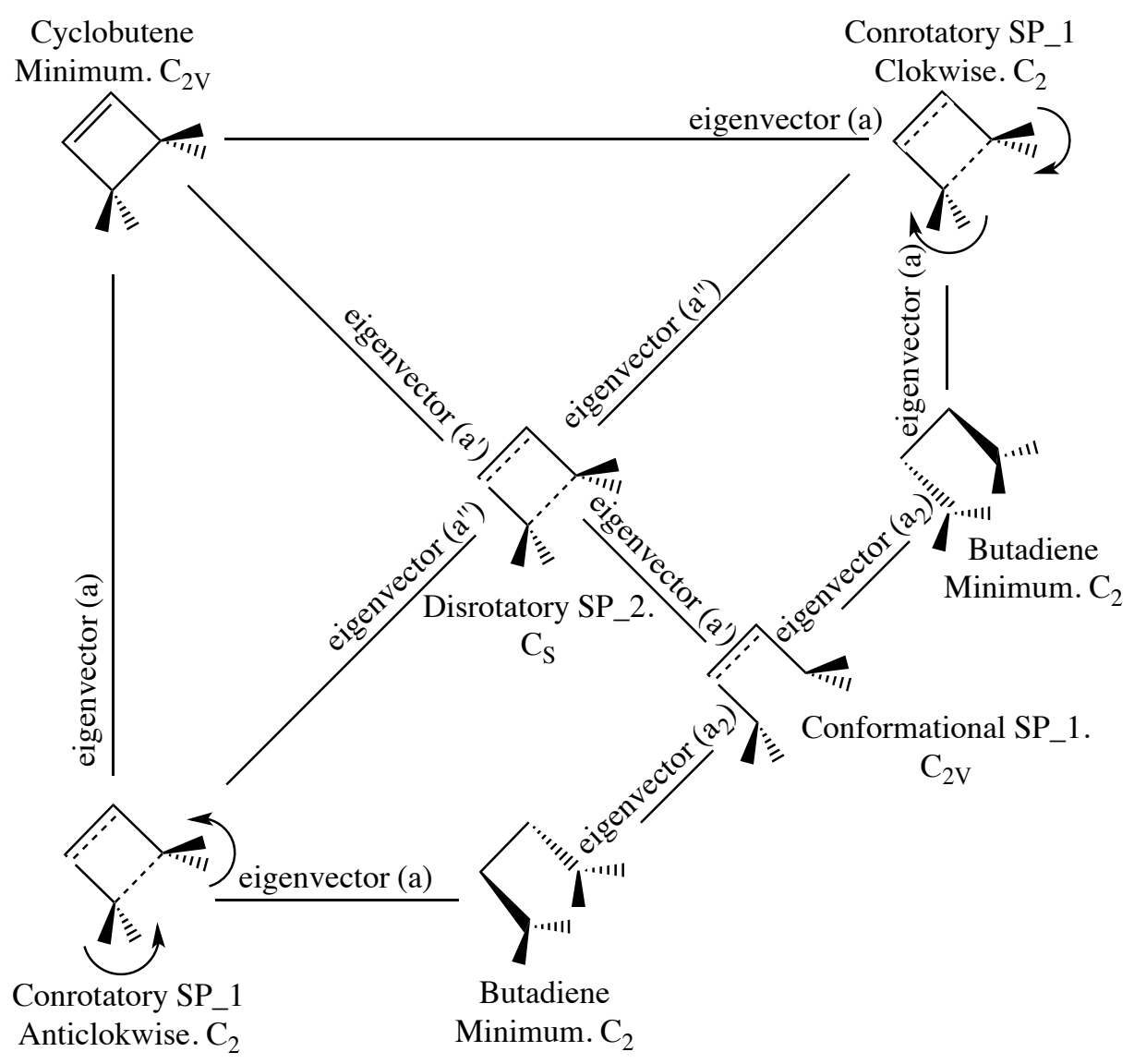

Figure 16: 2-dimensional cartoon of the ring opening of cyclobutene to cis-butadiene. Scheme for different pathways from the disrotatory $\mathrm{SP}_{2}$ in the center. The leading directions are eigenvectors of the Hessian to different symmetries.

PES such that it has $\mathrm{C}_{2}$ symmetry any procedure will move to a node of the PES of $\mathrm{C}_{s}$ symmetry. The wave function solution for the Schrödinger equation is symmetrically adapted.

The results are interesting in the sense that using the $\mathrm{C}_{1}$ symmetry approximation to $\mathrm{SP}_{2}$, the $\mathrm{NT}$ follows the eigenvector belonging to the a" reducible representation, and it converges to the first index saddle point $\left(\mathrm{SP}_{1}\right)$ on the cis-butadiene minimum energy path. Regarding the structure of the wave function a reminiscent butadiene electronic structure emerges. The highest occupied natural molecular orbital of the CAS space is the positive combination of the two $\pi$ bonds corresponding each to the "two ethenes" of the butadiene, in other words, $\pi\left(C_{4}-C_{1}\right)+\pi\left(C_{2}-C_{1}\right)$, the second is $\pi\left(C_{4}-C_{1}\right)-\pi\left(C_{2}-C_{1}\right)$, the third is $\pi^{*}\left(C_{4}-C_{1}\right)+\pi^{*}\left(C_{2}-C_{1}\right)$ and the fourth is $\pi^{*}\left(C_{4}-C_{1}\right)-\pi^{*}\left(C_{2}-C_{1}\right)$ which is the lowest occupied orbital. Now following the NT algorithm but starting at the approximated $\mathrm{SP}_{2}$ with $\mathrm{C}_{1}$ symmetry, using the eigenvector belonging to the a' reducible representation, this path converges to the cyclobutene. There are also the two reported VRI points. 
There is another pathway on the PES of cyclobutene not observed in this work. A totally symmetric way for an ascent from cyclobutene leads to the $\mathrm{SP}_{1}$ for a simultaneous dissociation of two H-atoms forming an $\mathrm{H}_{2}{ }^{76}$ In this reference, in contrast to the gritty realism of our work, no symmetryforbidden disrotatory reaction channel was found within the PES of the cyclobutene1,3-butadiene system. Instead of this, the disrotatory rotation of two methylene groups of cyclobutene results in the elimination of a molecule of dihydrogen giving rise to the formation of a low-stable $\pi$-complex $\mathrm{H}_{2} \cdot$ cyclobutadiene. But the TS of this process is much higher than our disrotatory $\mathrm{SP}_{2}$.

\section{ACKNOWLEDGMENTS}

Financial support from the Spanish Ministerio de Ciencia e Innovación, DGI project CTQ2011-22505 and, in part from the Generalitat de Catalunya projects 2014SGR-0139 is fully acknowledged.

The authors disclose any potential conflict of interest.

\section{Appendix}

Part of the scratch file for the communication of the GAD, the DOP $853,{ }^{58}$ and the GamessUS ${ }^{59}$ parts of the calculation. Note: the output file for an MCSCF calculation is really complicated and it sometimes changes according to many reasons; so it is not standard for UHF, RHF or GVB calculations. This is due to the complexity of MCSCF functional energy. So, one should sometimes control the usefulnes of the operations used in the scrach file.

$\cdots$

export status $=2$

while (test \$status -eq 2) do

./ProgramDOP

cat mcscf point ReadMOs > input

rm PUNCH

rm IRCDATA

firefly > output

grep -A164 'NATURAL' PUNCH > ReadMOs

grep -A1 'INTERNAL COORD HESSIAN' PUNCH > Energy

grep -A8 'TRANG: TRANSFORMED GRADIENT' output> Grad

grep -A137 'HESSIAN MATRIX IN INTERNAL' output > Hess

grep -A274 'GINVR: G MATRIX' output > Gmat

./ReadOut

export status $=\$$ ?

done

\# end of the GAD cycle

The status-variable controls the exit from the program if GAD is converged, or if an error-stop happens. The file mcscf contains the same head of the GamessUS input file for every point, but point is the current point in internal coordinates. The file ReadMOs is used in the next step to recycle the former optimized orbitals. The program ReadOut uses the current files with energy, gradient, Hessian, and metric matrices to translate these values back for input files of the ProgramDOP. In a second part of the program ReadOut the differential equations of the GAD ansatz are used to calculate the current tangent vector for the DOP853 routine. Jump parameters in the ProgramDOP save the interplay between steplength search, and the jump to the next point of the GAD trajectory. 


\section{References}

1. Wigner, E. P. Comm Pure Appl Math 1960, 13,1 .

2. Mezey, P. Potential Energy Hypersurfaces; Elsevier, Amsterdam, 1987.

3. Heidrich, D.; Kliesch, W.; Quapp, W. Properties of Chemically Interesting Potential Energy Surfaces; Lecture Notes in Chemistry 56 Springer, Berlin Heidelberg, 1991.

4. Wales, D. J. Energy Landscapes; Cambridge University Press, Cambridge, 2003.

5. Heidrich, D., Ed.; The Reaction Path in Chemistry: Current Approaches and Perspektives; Kluwer Academic Publishers, Dordrecht, 1995.

6. Kraka, E. Encyclopedia of computational chemistry; Schleyer, P. v. R., Ed.; Wiley, New York, 1998; p. 2437.

7. Pomerantz, A. E. J Am Chem Soc 2005, 127, 16368.

8. Suits, A. G. Acc Chem Res 2008, 41, 873 .

9. Heazlewood, B. R.; Jordan, M. J. T.; Kable, S. H.; Selby, T. M.; Osborn, D. L.; Shepler, B. C.; Braams, B. J.; Bowman, J. M. Proc Natl Acad Sci USA 2008, 105, 12719.

10. Ezra, G. S.; Wiggins, S. J Phys A 2009, 42, 205101.

11. Lu, Z.; Chang, Y.; Yin, Q.-Z.; Ng, C.; Jackson, W. Science 2014, 346, 61.

12. Heidrich, D.; Quapp, W. Theor Chim Acta 1986, 70, 89 .

13. Minyaev, R. M.; Getmanskii, I. V.; Quapp, W. Russ J Phys Chem 2004, 78, 1494.

14. Collins, P.; Carpenter, B.; Ezra, G.; Wiggins, S. J Chem Phys 2013, 139, 154108.

15. E, W.; Zhou, X. Nonlinearity 2011, 24, 1831.
16. Quapp, W.; Hirsch, M.; Imig, O.; Heidrich, D. J Comput Chem 1998, 19, 1087.

17. Quapp, W.; Hirsch, M.; Heidrich, D. Theor Chem Acc 1998, 100, 285.

18. Quapp, W. J Theoret Comput Chem 2003, 2,385 .

19. Fukui, K. J Phys Chem 1970, 74, 4161.

20. Quapp, W.; Heidrich, D. Theor Chim Acta 1984, 66, 245.

21. Crippen, G. M.; Scheraga, H. A. Arch Biochem BioPhys 1971, 144, 462 .

22. Bofill, J. M.; Quapp, W.; Caballero, M. Chem Phys Lett 2013, 583, 203.

23. Sun, J.-Q.; Ruedenberg, K. J Chem Phys 1994, 101, 2157.

24. Li, T.; Zhang, P.; Zhang, W. Multiscale Model Simul 2013, 11, 385.

25. Samanta, A.; Chen, M.; Yu, T.-Q.; Tuckerman, M.; E, W. J Chem Phys 2014, 140, 164109 .

26. Zeng, Y.; Xiao, P.; Henkelman, G. J Chem Phys 2014, 140, 044115.

27. Lanczos, C. J Res Nat Bureau Standards 1950, 45, 255 .

28. Lanczos, C. J Res Nat Bureau Standards 1952, 49, 33 .

29. Brown, P. N.; Saad, Y. SIAM J Sci Stat Comput 1990, 11, 450.

30. Fokkema, D. R.; Sleijpen, G. L. G.; Van der Vorst, H. A. SIAM J Sci Stat Comput 1998, 19,657 .

31. Quapp, W.; Bofill, J. M.; Bernuz, E. J Math Chem 2015, 53, 41 .

32. Quapp, W.; Bofill, J. M. Theor Chem Acc 2014, 133, 1510. 
33. Samanta, A.; E, W. J Chem Phys 2012, 136, 124104.

34. Tachibana, A. Theoret Chim Acta 1981, 58, 301 .

35. Khait, Y. G.; Panin, A. I.; Averyanov, A. S. Int J Quantum Chem 1994, 54, 329.

36. Bartsch, T.; Uzer, T. J Phys B 2005, 38, 241.

37. Minyaev, R. M.; Quapp, W.; Schmidt, B.; Getmanski, I. V.; Koval, V. V. Chem Phys 2013, 425, 170 .

38. Mauguière, F. A.; Collins, P.; Ezra, G. S.; Wiggins, S. J Chem Phys 2013, 138, 134118.

39. Mauguière, F. A.; Collins, P.; Ezra, G. S.; Farantos, S. C.; Wiggins, S. J Chem Phys 2014, 140, 134112.

40. Nagahata, Y.; Teramoto, H.; Li, C. B.; Kawai, S.; Komatsuzaki, T. Phys Rev E Stat Nonlin Soft Matter Phys 2013, 88, 042923.

41. Parlett, B. The symmetric eigenvalue problem; Prentice-Hall, Englewood Cliffs, NJ, 1980.

42. Williams, I.; Maggiora, G. J Mol Struct (Theochem) 1982, 89, 356.

43. Quapp, W.; Hirsch, M.; Heidrich, D. Theor Chem Acc 1998, 100, 285.

44. Quapp, W.; Hirsch, M.; Imig, O.; Heidrich, D. J Comput Chem 1998, 19, 1087.

45. Quapp, W. J Comput Chem 2001, 22, 537 .

46. Hirsch, M.; Quapp, W. J Comput Chem $2002,23,887$.

47. Anglada, J. M.; Besalú, E.; Bofill, J. M.; Crehuet, R. J Comput Chem 2001, 22, 387.

48. Bofill, J. M.; Anglada, J. M. Theor Chem Acc 2001, 105, 463.

49. Crehuet, R.; Bofill, J. M.; Anglada, J. M. Theor Chem Acc 2002, 107, 130.
50. Quapp, W. J Molec Struct 2004, 695-696, 95.

51. Bofill, J.; Quapp, W. J Chem Phys 2011, 134, 074101.

52. Branin, F. H. IBM J Res Develop 1972, 16, 504.

53. Quapp, W. J Math Chem 2004, 36, 365 .

54. Hirsch, M.; Quapp, W. J Math Chem 2004, 36,307 .

55. Diener, I. Globale Aspekte des kontinuierlichen Newtonverfahrens; Göttingen, 1991. Habilitation.

56. Hirsch, M. Dissertation (Ph.D.Thesis) ; Universität Leipzig, Germany, 2004.

57. Quapp, W.; Schmidt, B. Theor Chem Acc $2011,128,47$.

58. Hairer, E.; Norsett, S.; Wanner, G. Solving Ordinary Differential Equations I. Nonstiff Problems, 2nd Ed.; Series Computat. Math. Springer, 1993.

59. Schmidt, M. W.; Baldridge, K. K.; Boatz, J. A.; Elbert, S. T.; Gordon, M. S.; Jensen, J. H.; Koseki, S.; Matsunaga, N.; Nguyen, K. A.; Su, S.; Windus, T. L.; Dupuis, M.; Montgomery, J. A. J Comput Chem 1993, 14,1347 .

60. Wolfram Mathematica10; Wolfram Research, Inc., Champaign, 2015.

61. Schaftenaar, G. Molden5; CAOS/CAMM Center, Nijmegen, 2008.

62. Gordon, M. S.; Schmidt, M. W. Theory and Applications of Computational Chemistry: the first forty years; Dykstra, C.; Frenking, G.; Kim, K.; Scuseria, G., Eds.; Elsevier, Amsterdam, 2005; p. 1167 .

63. Granovsky, A. A. Firefly Version 7.1.G. http://classic.chem.msu.su/gran/firefly/index.html. 
64. McWeeny, R. Methods of Molecular Quantum Mechanics; Academic Press, San Diego, 1989.

65. Dill, J. D.; Pople, J. A. J Chem Phys 1975, 62, 2921 .

66. Cooper, W.; Walters, W. D. J Am Chem Soc 1958, 80, 4220 .

67. Vogel, E. Liebigs Ann Chem 1958, 615, 14.

68. Criegee, R.; Noll, K. Liebigs Ann Chem 1959, 627, 1 .

69. Criegee, R.; Seebach, D.; Winter, R. E.; Borretzen, B.; Bunter, H.-A. Chem Ber 1965, 98, 2339 .

70. Srinivasan, R. J Am Chem Soc 1969, 91, 7557.

71. Woodward, R. B.; Hoffmann, R. J Am Chem Soc 1965, 87, 395.

72. Woodward, R. B.; Hoffmann, R. Angew Chem Int Ed Engl 1969, 8, 781.

73. Longuet-Higgins, H. C.; Abrahamson, E. W. J Am Chem Soc 1965, 87, 2045.

74. Houk, K. N.; Li, Y.; Evanseck, J. D. Angew Chem Int Ed Engl 1992, 31, 682.

75. W. R. Dolbier, J.; Koroniack, H.; Houk, K. N.; Sheu, C. Acc Chem Res 1996, 29, 471.

76. Koval, V. V.; Minyaev, R. M.; Minkin, V. I. J Computat Theoret Chem 2014, 1030, 44 .

77. Dewar, M. J. S. J Am Chem Soc 1952, 74, 3341, 3345, 3350, 3353, 3355, 3357.

78. Dewar, M. J. S. Tetrahedron Suppl B 1966, , 76 .

79. Zimmerman, H. E. J Am Chem Soc 1966, 88, 1564 .

80. Breulet, J.; Schaefer III, H. F. J Am Chem Soc 1984, 106, 1221.
81. van der Lugt, W. T. A. M.; Oosterhoff, L. J. J Am Chem Soc 1969, 91, 6042.

82. Grimbert, D.; Segal, G.; Devaquet, A. J Am Chem Soc 1975, 97, 6629.

83. Hirsch, M.; Quapp, W. J Molec Struct, THEOCHEM 2004, 683, 1.

84. Crehuet, R.; Bofill, J. M. J Chem Phys 2005, $122,234105$.

85. Quapp, W.; Bofill, J. M.; Caballero, M. Chem Phys Lett 2012, 541, 122.

86. Ben-Nun, M.; Martínez, T. J. J Am Chem Soc 2000, 122, 6299 .

87. Hirsch, M.; Quapp, W.; Heidrich, D. Phys Chem Chem Phys 1999, 1, 5291.

88. Quapp, W.; Melnikov, V. Phys Chem Chem Phys 2001, 3, 2735.

89. Bofill, J. Dissertation (Ph.D. Thesis); Universitat de Barcelona, 1987.

90. Baker, J.; Muir, M.; Andzehn, J. J Chem Phys 1988, 102, 2063.

91. Bell, R. P. Proc R Soc London, Ser A 1936, 154,414 .

92. Evans, M. G.; Polanyi, M. Trans Faraday Soc 1938, 34, 11 .

93. Evans, M. G.; Warhurst, E. Trans Faraday Soc 1938, 34, 614 .

94. Shaik, S. S.; Schlegel, H. B.; Wolfe, S. Theoretical Aspects of Physical Organic Chemistry. The $S_{N} 2$ Mechanism.; John Wiley \& Sons, Inc., New York, 1992. 